\title{
Singular Reduction Operators in Two Dimensions
}

\author{
Michael KUNZINGER ${ }^{\dagger}$ and Roman O. POPOVYCH ${ }^{\ddagger}$ \\ ${ }^{\dagger,}$ Fakultät für Mathematik, Universität Wien, Nordbergstraße 15, A-1090 Wien, Austria \\ $\ddagger$ Institute of Mathematics of NAS of Ukraine, 3 Tereshchenkivska Str., Kyiv-4, Ukraine \\ E-mail: ${ }^{\dagger}$ michael.kunzinger@univie.ac.at, ${ }^{\ddagger}$ rop@imath.kiev.ua
}

The notion of singular reduction operators, i.e., of singular operators of nonclassical (conditional) symmetry, of partial differential equations in two independent variables is introduced. All possible reductions of these equations to first-order ODEs are are exhaustively described. As examples, properties of singular reduction operators of $(1+1)$-dimensional evolution and wave equations are studied. It is shown how to favourably enhance the derivation of nonclassical symmetries for this class by an in-depth prior study of the corresponding singular vector fields.

\section{Introduction}

Distinctions in kind between Lie symmetries and nonclassical symmetries became apparent already in the first presentation of nonclassical symmetries in [3] by the example of the $(1+1)$ dimensional linear heat equation and a particular class of operators. In contrast to classical Lie symmetries (see, e.g., [17]), the system of determining equations on the coefficients of nonclassical symmetry operators of the heat equation was found to be nonlinear and less overdetermined, and the set of such operators does not possess the structure of an algebra or even a vector space.

Another difference appears in the procedure of deriving the determining equations. Namely, deriving systems of determining equations for nonclassical symmetries crucially depends on the interplay between the operators and the equations under consideration. Thus, for the linear heat equation $u_{t}=u_{x x}$ the general form of nonclassical symmetry operators is $Q=\tau(t, x, u) \partial_{t}+$ $\xi(t, x, u) \partial_{x}+\eta(t, x, u) \partial_{u}$, where $(\tau, \xi) \neq(0,0)$, and there are two essentially different cases of nonclassical symmetries: the regular case $\tau \neq 0$ and the singular case $\tau=0$. The factorization up to nonvanishing functional multipliers gives the two respective cases for the further investigation: 1) $\tau=1$ and 2) $\tau=0, \xi=1$.

The problem of determining the nonclassical symmetries of the linear heat equation was completely solved in [10]. In the regular case $\tau=1$, after partial integration of the corresponding determining equations, we obtain $\xi=g^{1}(t, x)$ and $\eta=g^{2}(t, x) u+g^{3}(t, x)$. The functions $g^{1}, g^{2}$ and $g^{3}$ satisfy a coupled nonlinear system of partial differential equations [3], which is linearized by a nonlocal transformation to a system of three uncoupled copies of the initial equation [9, 10, 24]. The underlying reason for this phenomenon lies in the interaction between the linearity and the evolution structure in the linear heat equation. Hence similar results can be obtained only for linear evolution equations [7, 18, 21] or related linearizable equations [13].

The singular case $(\tau, \xi)=(0,1)$ was not considered in [3]. In this case the system of determining equations for nonclassical symmetries consists of a single $(1+2)$-dimensional nonlinear evolution equation for the unknown function $\eta$ and, therefore, is not overdetermined. The determining equation is reduced by a nonlocal transformation to the initial equation with an additional implicit independent variable which can be assumed as a parameter [10]. The linearity of the heat equation is inessential here. Hence after the case of linear evolutions equations [7, 18] this result was extended to general $(1+1)$-dimensional evolution equations [25], multi-dimensional evolution equations [19] and even systems of such equations [23]. Moreover, it was proved [19], that, e.g., in the $(1+1)$-dimensional case there exists a one-to-one correspondence between one-parametric families of solutions of an evolution equation and its reduction operators with $(\tau, \xi)=(0,1)$. 
The above results raise a number of interesting questions, to wit: What are possible causes for the existence of singular cases for reduction operators? Is the conventional partition of sets of reduction operators with the conditions of vanishing and nonvanishing coefficients of operators universal or is it appropriate only for certain classes of differential equations, e.g., evolution equations? Can partitions of sets of reduction operators, different from the conventional one, be useful? Does there exist an algorithmic way of singling out singular cases for reduction operators before deriving determining equations? What properties of a partial differential equation and a subset of its reduction operators lead to a 'no-go' situation (i.e., a single determining equation equivalent, in a certain sense, to the initial equation)? What is the optimal way of obtaining the determining equation for nonclassical symmetries? The purpose of the present paper is to answer these and other related questions.

Algorithms for deriving the determining equations for nonclassical symmetries were discussed, e.g., in [2, 6] but the focus of these works was quite different.

The conditional invariance of a differential equation with respect to an operator is equivalent to any ansatz associated with this operator reducing the equation to a differential equation with one less independent variables [26]. That is why we use the shorter and more natural term "reduction operators" instead of "operators of conditional symmetry" or "operators of nonclassical symmetry" and say that an operator reduces a differential equation if the equation is reduced by the corresponding ansatz. The direct method of reduction with ansatzes of a special form was first explicitly applied in [4] to the Boussinesq equation although reductions by non-Lie ansatzes were already discussed, e.g., in [8. A connection between the reduction by generalized ansatzes and compatibility with respect to higher-order constraints was found in [15].

To clarify the main ideas of the proposed framework of singular reduction operators, in this first presentation of the subject we consider only the case of a single partial differential equation in two independent and one dependent variables and a single reduction operator. We note, however, that more general cases can be included and will be the subject of forthcoming papers.

Some of the main conclusions of the present paper are:

- Singular cases of reduction operators of a partial differential equation are connected with the possibility of lowering the order of this equation on the manifolds determined by the corresponding invariant surface conditions in the appropriate jet space. Hence the first step of the procedure of finding nonclassical symmetries has to consist in studying singular modules of vector fields which lower the order of the equation. This step is entirely algorithmic, hence is especially suited to a direct implementation in symmetryfinding computer algebra programs. The structure of singular modules of vector fields has to be taken into account under splitting the set of reduction operators for factorization.

- The weak singularity co-order of a reduction operator $Q$ coincides with the essential order of the corresponding reduced equation and the number of essential parameters in the family of $Q$-invariant solutions.

- If a single partial differential equation $\mathcal{L}$ in two independent variables admits a first coorder singular module $S$ of vector fields then it necessarily possesses first co-order singular reduction operators belonging to $S$. The system of determining equations for such operators consists of a single partial differential equation $\mathrm{DE}$ in three independent variables of the same order as $\mathcal{L}$. The equation $\mathrm{DE}$ is reduced to $\mathcal{L}$ by a nonlocal transformation.

The paper is organized as follows: The main notions and statements on nonclassical symmetries are presented in Section 2, Singular vector fields of differential functions and differential equations are defined and studied in Sections 3 and 4 , respectively. Singular reduction operators of $(1+1)$-dimensional evolution and nonlinear wave equations are exhaustively investigated in 
Sections 5 and 6. It is shown that the conventional partition of sets of reduction operators is natural for evolution equations, in contrast to the case of nonlinear wave equations. A connection between the singularity co-order of reduction operators and the number of parameters in the corresponding families of invariant solutions is established in Section 7 . The final Section 8 is devoted to first co-order singular reduction operators of general partial differential equations in two independent and one dependent variables.

\section{Reduction operators of differential equations}

Following [11, 12, 22, 26], in this section we briefly collect the required notions and results on nonclassical (conditional) symmetries of differential equations. Also, we argue for the use of the name "reduction operators" instead of "nonclassical (conditional) symmetry operators". In accordance with the aims of this paper we restrict our considerations to the case of two independent variables and a single reduction operator.

The set of (first-order) differential operators (or vector fields) of the general form

$$
Q=\xi^{i}(x, u) \partial_{i}+\eta(x, u) \partial_{u}, \quad\left(\xi^{1}, \xi^{2}\right) \neq(0,0),
$$

will be denoted by $\mathfrak{Q}$. In what follows, $x$ denotes the pair of independent variables $\left(x_{1}, x_{2}\right)$ and $u$ is treated as the unknown function. The index $i$ runs from 1 to 2 , and we use the summation convention for repeated indices. Subscripts of functions denote differentiation with respect to the corresponding variables, $\partial_{i}=\partial / \partial x_{i}$ and $\partial_{u}=\partial / \partial u$. Any function is considered as its zero-order derivative. All our considerations are carried out in the local setting.

Two differential operators $\widetilde{Q}$ and $Q$ are called equivalent if they differ by a multiplier which is a non-vanishing function of $\underset{\widetilde{Q}}{x}$ and $u$ : $\widetilde{Q}=\lambda Q$, where $\lambda=\lambda(x, u), \lambda \neq 0$. The equivalence of operators will be denoted by $\widetilde{Q} \sim Q$. Factoring $\mathfrak{Q}$ with respect to this equivalence relation we arrive at $\mathfrak{Q}_{\mathrm{f}}$. Elements of $\mathfrak{Q}_{\mathrm{f}}$ will be identified with their representatives in $\mathfrak{Q}$.

The first-order differential function

$$
Q[u]:=\eta(x, u)-\xi^{i}(x, u) u_{i}
$$

is called the characteristic of the operator $Q$. The characteristic $\operatorname{PDE} Q[u]=0$ (also known as the invariant surface condition) has two functionally independent solutions $\zeta(x, u)$ and $\omega(x, u)$. Therefore, the general solution of this equation can be implicitly represented in the form $F(\zeta, \omega)=0$, where $F$ is an arbitrary function.

A differential function $\Theta=\Theta[z]$ of the dependent variables $z=\left(z^{1}, \ldots, z^{m}\right)$ which in turn are functions of a tuple of independent variables $y=\left(y_{1}, \ldots, y_{n}\right)$ will be considered as a smooth function of $y$ and derivatives of $z$ with respect to $y$. The order $r=\operatorname{ord} \Theta$ of the differential function $\Theta$ equals the maximal order of derivatives involved in $\Theta$. More precisely, the differential function $\Theta$ is defined as a function on a subset of the jet space $J^{r}(y \mid z)$ [14].

The characteristic equations of equivalent operators have the same set of solutions. Conversely, any family of two functionally independent functions of $x$ and $u$ is a complete set of integrals of the characteristic equation of a differential operator. Therefore, there exists a one-to-one correspondence between $\mathfrak{Q}_{\mathrm{f}}$ and the set of families of two functionally independent functions of $x$ and $u$, which is factorized with respect to the corresponding equivalence relation. (Two families of the same number of functionally independent functions of the same arguments are considered equivalent if any function from one of the families is functionally dependent on functions from the other family.)

Since $\left(\xi^{1}, \xi^{2}\right) \neq(0,0)$ we can assume without loss of generality that $\zeta_{u} \neq 0$ and $F_{\zeta} \neq 0$ and resolve the equation $F=0$ with respect to $\zeta: \zeta=\varphi(\omega)$. This implicit representation of the function $u$ is called an ansatz corresponding to the operator $Q$. 
Consider an $r$ th order differential equation $\mathcal{L}$ of the form $L\left(x, u_{(r)}\right)=0$ for the unknown function $u$ of two independent variables $x=\left(x_{1}, x_{2}\right)$. Here $L=L[u]=L\left(x, u_{(r)}\right)$ is a fixed differential function of order $r$ and $u_{(r)}$ denotes the set of all the derivatives of the function $u$ with respect to $x$ of order not greater than $r$, including $u$ as the derivative of order zero. Within the local approach the equation $\mathcal{L}$ is treated as an algebraic equation in the jet space $J^{r}=J^{r}(x \mid u)$ of order $r$ and is identified with the manifold of its solutions in $J^{r}$ :

$$
\mathcal{L}=\left\{\left(x, u_{(r)}\right) \in J^{r} \mid L\left(x, u_{(r)}\right)=0\right\} .
$$

Denote the manifold defined by the set of all the differential consequences of the characteristic equation $Q[u]=0$ in $J^{r}$ by $\mathcal{Q}_{(r)}$, i.e.,

$$
\mathcal{Q}_{(r)}=\left\{\left(x, u_{(r)}\right) \in J^{r} \mid D_{1}^{\alpha} D_{2}^{\beta} Q[u]=0, \alpha, \beta \in \mathbb{N} \cup\{0\}, \alpha+\beta<r\right\},
$$

where $D_{1}=\partial_{1}+u_{\alpha+1, \beta} \partial_{u_{\alpha \beta}}$ and $D_{2}=\partial_{2}+u_{\alpha, \beta+1} \partial_{u_{\alpha \beta}}$ are the operators of total differentiation with respect to the variables $x_{1}$ and $x_{2}$, and the variable $u_{\alpha \beta}$ of the jet space $J^{r}$ corresponds to the derivative $\partial^{\alpha+\beta} u / \partial x_{1}^{\alpha} \partial x_{2}^{\beta}$.

A precise and rigorous definition of nonclassical (or conditional) symmetry was first suggested in [11] (see also [12, 26]).

Definition 1. The differential equation $\mathcal{L}$ is called conditionally invariant with respect to the operator $Q$ if the relation $\left.Q_{(r)} L\left(x, u_{(r)}\right)\right|_{{\mathcal{L} \cap \mathcal{Q}^{(r)}}=0 \text { holds, which is called the conditional }}$ invariance criterion. Then $Q$ is called an operator of conditional symmetry (or $Q$-conditional symmetry, nonclassical symmetry, etc.) of the equation $\mathcal{L}$.

In Definition 1 the symbol $Q_{(r)}$ stands for the standard $r$ th prolongation of the operator $Q$ [14, 17]:

$$
Q_{(r)}=Q+\sum_{0<\alpha+\beta \leqslant r} \eta^{\alpha \beta} \partial_{u_{\alpha \beta}}, \quad \eta^{\alpha \beta}:=D_{1}^{\alpha} D_{2}^{\beta} Q[u]+\xi^{1} u_{\alpha+1, \beta}+\xi^{2} u_{\alpha, \beta+1} .
$$

The equation $\mathcal{L}$ is conditionally invariant with respect to $Q$ if and only if the ansatz $\zeta=\varphi(\omega)$ constructed with $Q$ reduces $\mathcal{L}$ to an ordinary differential equation $\check{\mathcal{L}}: \check{L}[\varphi]=0$ [26]. Namely, there exist differential functions $\check{\lambda}=\check{\lambda}[\varphi]$ and $\check{L}=\check{L}[\varphi]$ of an order not greater than $r$ (i.e., functions of $\omega$ and derivatives of $\varphi$ with respect to $\omega$ up to order $r)$ such that $\left.L\right|_{u=\varphi(\omega)}=\check{\lambda} \check{L}$. The function $\check{\lambda}$ does not vanish and may depend on $\theta$ as a parameter, where the value $\theta=\theta(x, u)$ is functionally independent of $\zeta$ and $\omega$. The differential function $\check{L}$ is assumed to be of minimal order $\check{r}$ which is possibly reached up to the equivalence generated by nonvanishing multipliers. Then the reduced equation $\check{\mathcal{L}}$ is of essential order $\check{r}$.

This is why we will also call operators of conditional symmetry reduction operators of $\mathcal{L}$.

Another treatment of conditional invariance is that the system $\mathcal{L} \cap \mathcal{Q}_{(r)}$ is compatible in the sense of not involving any nontrivial differential consequences [15, 16].

The property of conditional invariance is compatible with the equivalence relation on $\mathfrak{Q}$ [12, 26]:

Lemma 1. If the equation $\mathcal{L}$ is conditionally invariant with respect to the operator $Q$ then it is conditionally invariant with respect to any operator which is equivalent to $Q$.

The set of reduction operators of the equation $\mathcal{L}$ is a subset of $\mathfrak{Q}$ and so will be denoted by $\mathfrak{Q}(\mathcal{L})$. In view of Lemma 1, $Q \in \mathfrak{Q}(\mathcal{L})$ and $\widetilde{Q} \sim Q$ imply $\widetilde{Q} \in \mathfrak{Q}(\mathcal{L})$, i.e., $\mathfrak{Q}(\mathcal{L})$ is closed under the equivalence relation on $\mathfrak{Q}$. Therefore, the factorization of $\mathfrak{Q}$ with respect to this equivalence relation can be naturally restricted to $\mathfrak{Q}(\mathcal{L})$, resulting in the subset $\mathfrak{Q}_{\mathrm{f}}(\mathcal{L})$ of $\mathfrak{Q}_{\mathrm{f}}$. As in the whole set $\mathfrak{Q}_{\mathrm{f}}$, we identify elements of $\mathfrak{Q}_{\mathrm{f}}(\mathcal{L})$ with their representatives in $\mathfrak{Q}(\mathcal{L})$. In this approach the problem of completely describing all reduction operators for $\mathcal{L}$ is equivalent to finding $\mathfrak{Q}_{\mathrm{f}}(\mathcal{L})$.

The conditional invariance criterion admits the following useful reformulation [26]. 
Lemma 2. Given a differential equation $\mathcal{L}: L[u]=0$ of order $r$ and differential functions $\tilde{L}[u]$ and $\lambda[u] \neq 0$ of an order not greater than $r$ such that $\left.L\right|_{\mathcal{Q}_{(r)}}=\left.\lambda \tilde{L}\right|_{\mathcal{Q}_{(r)}}$, an operator $Q$ is a reduction operator of $\mathcal{L}$ if and only if the relation $\left.Q_{(\tilde{r})} \tilde{L}\right|_{\tilde{\mathcal{L}} \cap \mathcal{Q}_{(\tilde{r})}}=0$ holds, where $\tilde{r}=$ ord $\tilde{L} \leqslant r$ and the manifold $\tilde{\mathcal{L}}$ is defined in $J^{\tilde{r}}$ by the equation $\tilde{L}[u]=0$.

The classification of reduction operators can be considerably enhanced and simplified by considering Lie symmetry and equivalence transformations of (classes of) equations.

Lemma 3. Any point transformation of $x$ and $u$ induces a one-to-one mapping of $\mathfrak{Q}$ into itself. Namely, the transformation $g: \tilde{x}_{i}=X^{i}(x, u), \tilde{u}=U(x, u)$ generates the mapping $g_{*}: \mathfrak{Q} \rightarrow \mathfrak{Q}$ such that the operator $Q$ is mapped to the operator $g_{*} Q=\tilde{\xi}^{i} \partial_{\tilde{x}_{i}}+\tilde{\eta} \partial_{\tilde{u}}$, where $\tilde{\xi}^{i}(\tilde{x}, \tilde{u})=Q X^{i}(x, u)$, $\tilde{\eta}(\tilde{x}, \tilde{u})=Q U(x, u)$. If $Q^{\prime} \sim Q$ then $g_{*} Q^{\prime} \sim g_{*} Q$. Therefore, the corresponding factorized mapping $g_{\mathrm{f}}: \mathfrak{Q}_{\mathrm{f}} \rightarrow \mathfrak{Q}_{\mathrm{f}}$ also is well defined and bijective.

Definition $2([20])$. Differential operators $Q$ and $\widetilde{Q}$ are called equivalent with respect to a group $G$ of point transformations if there exists $g \in G$ for which the operators $Q$ and $g_{*} \widetilde{Q}$ are equivalent. We denote this equivalence by $Q \sim \widetilde{Q} \bmod G$.

Lemma 4. Given any point transformation $g$ of an equation $\mathcal{L}$ to an equation $\tilde{\mathcal{L}}, g_{*}$ maps $\mathfrak{Q}(\mathcal{L})$ to $\mathfrak{Q}(\tilde{\mathcal{L}})$ bijectively. The same is true for the factorized mapping $g_{\mathrm{f}}$ from $\mathfrak{Q}_{\mathrm{f}}(\mathcal{L})$ to $\mathfrak{Q}_{\mathrm{f}}(\tilde{\mathcal{L}})$.

Corollary 1. Let $G$ be the point symmetry group of an equation $\mathcal{L}$. Then the equivalence of operators with respect to the group $G$ generates equivalence relations in $\mathfrak{Q}(\mathcal{L})$ and in $\mathfrak{Q}_{\mathrm{f}}(\mathcal{L})$.

Consider the class $\left.\mathcal{L}\right|_{\mathcal{S}}$ of equations $\mathcal{L}_{\theta}: L\left(x, u_{(r)}, \theta\right)=0$ parameterized with the parameterfunctions $\theta=\theta\left(x, u_{(r)}\right)$. Here $L$ is a fixed function of $x, u_{(r)}$ and $\theta$. The symbol $\theta$ denotes the tuple of arbitrary (parametric) differential functions $\theta\left(x, u_{(r)}\right)=\left(\theta^{1}\left(x, u_{(r)}\right), \ldots, \theta^{k}\left(x, u_{(r)}\right)\right)$ running through the set $\mathcal{S}$ of solutions of the system $S\left(x, u_{(r)}, \theta_{(q)}\left(x, u_{(r)}\right)\right)=0$. This system consists of differential equations on $\theta$, where $x$ and $u_{(r)}$ play the role of independent variables and $\theta_{(q)}$ stands for the set of all the derivatives of $\theta$ of order not greater than $q$. In what follows we call the functions $\theta$ arbitrary elements. Denote the point transformation group preserving the form of the equations from $\left.\mathcal{L}\right|_{\mathcal{S}}$ by $G^{\sim}$.

Let $P$ denote the set of the pairs consisting of an equation $\mathcal{L}_{\theta}$ from $\left.\mathcal{L}\right|_{\mathcal{S}}$ and an operator $Q$ from $\mathfrak{Q}\left(\mathcal{L}_{\theta}\right)$. In view of Lemma 4, the action of transformations from the equivalence group $G^{\sim}$ on $\left.\mathcal{L}\right|_{\mathcal{S}}$ and $\left\{\mathfrak{Q}\left(\mathcal{L}_{\theta}\right) \mid \theta \in \mathcal{S}\right\}$ together with the pure equivalence relation of differential operators naturally generates an equivalence relation on $P$.

Definition 3. Let $\theta, \theta^{\prime} \in \mathcal{S}, Q \in \mathfrak{Q}\left(\mathcal{L}_{\theta}\right), Q^{\prime} \in \mathfrak{Q}\left(\mathcal{L}_{\theta^{\prime}}\right)$. The pairs $\left(\mathcal{L}_{\theta}, Q\right)$ and $\left(\mathcal{L}_{\theta^{\prime}}, Q^{\prime}\right)$ are called $G^{\sim}$-equivalent if there exists $g \in G^{\sim}$ such that $g$ transforms the equation $\mathcal{L}_{\theta}$ to the equation $\mathcal{L}_{\theta^{\prime}}$, and $Q^{\prime} \sim g_{*} Q$.

The classification of reduction operators with respect to $G^{\sim}$ will be understood as the classification in $P$ with respect to this equivalence relation, a problem which can be investigated similar to the usual group classification in classes of differential equations. Namely, we construct firstly the reduction operators that are defined for all values of $\theta$. Then we classify, with respect to $G^{\sim}$, the values of $\theta$ for which the equation $\mathcal{L}_{\theta}$ admits additional reduction operators.

\section{$3 \quad$ Singular vector fields of differential functions}

Consider a vector field $Q=\xi^{i}(x, u) \partial_{i}+\eta(x, u) \partial_{u}$ with $\left(\xi^{1}, \xi^{2}\right) \neq(0,0)$, defined in the space $(x, u)$, and a differential function $L=L[u]$ of order ord $L=r$ (i.e., a smooth function of $x=\left(x_{1}, x_{2}\right)$ and derivatives of $u$ of orders up to $r$ ). 
Definition 4. The vector field $Q$ is called singular for the differential function $L$ if there exists a differential function $\tilde{L}=\tilde{L}[u]$ of an order less than $r$ such that $\left.L\right|_{\mathcal{Q}_{(r)}}=\left.\tilde{L}\right|_{\mathcal{Q}_{(r)}}$. Otherwise $Q$ is called a regular vector field for the differential function $L$. If the minimal order of differential functions whose restrictions on $\mathcal{Q}_{(r)}$ coincide with $\left.L\right|_{\mathcal{Q}_{(r)}}$ equals $k(k<r)$ then the vector field $Q$ is said to be of singularity co-order $k$ for the differential function $L$. The vector field $Q$ is called ultra-singular for the differential function $L$ if $\left.L\right|_{\mathcal{Q}_{(r)}} \equiv 0$.

For convenience, the singularity co-order of ultra-singular vector fields and the order of identically vanishing differential functions are defined to equal -1 . Regular vector fields for the differential function $L$ are defined to have singularity co-order $r=\operatorname{ord} L$. The singularity coorder of a vector field $Q$ for a differential function $L$ will be denoted by $\operatorname{sco}_{L} Q$.

If $Q$ is a singular vector field for $L$ then any vector field equivalent to $Q$ is singular for $L$ with the same co-order of singularity.

A function $\tilde{L}$ satisfying the conditions of Definition 4 can be constructively found. Namely, without loss of generality we can suppose that the coefficient $\xi^{2}$ of $\partial_{2}$ in $Q$ is nonzero. Then any derivative of $u$ of order not greater than $r$ can be expressed, on the manifold $\mathcal{Q}_{(r)}$, via derivatives of $u$ with respect to $x_{1}$ only. For example, for the first- and second-order derivatives we have

$$
\begin{aligned}
& u_{2}=\hat{\eta}-\hat{\xi} u_{1}, \\
& u_{12}=\hat{\eta}_{1}-\hat{\xi}_{1} u_{1}+\hat{\eta}_{u} u_{1}-\hat{\xi}_{u} u_{1}^{2}-\hat{\xi} u_{11}, \\
& u_{22}=\hat{\eta}_{2}-\hat{\xi}_{2} u_{1}+\left(\hat{\eta}_{u}-\hat{\xi}_{u} u_{1}\right)\left(\hat{\eta}-\hat{\xi} u_{1}\right)-\hat{\xi}\left(\hat{\eta}_{1}-\hat{\xi}_{1} u_{1}+\hat{\eta}_{u} u_{1}-\hat{\xi}_{u} u_{1}^{2}-\hat{\xi} u_{11}\right),
\end{aligned}
$$

where $\hat{\xi}=\xi^{1} / \xi^{2}$ and $\hat{\eta}=\eta / \xi^{2}$. After substituting the expressions for the derivatives into $L$, we obtain a differential function $\hat{L}$ depending only on $x, u$ and derivatives of $u$ with respect to $x_{1}$. We will call $\hat{L}$ a differential function associated with $L$ on the manifold $\mathcal{Q}_{(r)}$. The vector field $Q$ is singular for the differential function $L$ if and only if the order of $\hat{L}$ is less than $r$. The co-order of singularity of $Q$ equals the order of $\hat{L}$. The vector field $Q$ is ultra-singular if and only if $\hat{L} \equiv 0$. Therefore, testing that a vector field is singular for a differential function with two independent variables is realized in an entirely algorithmic procedure and can be easily included in existing programs for symbolic calculations of symmetries.

Consider the two-dimensional module $\left\{Q^{\theta}=\theta^{i} Q^{i}\right\}$ of vector fields over the ring of smooth functions of $(x, u)$ generated by the vector fields $Q^{i}=\xi^{i j}(x, u) \partial_{j}+\eta^{i}(x, u) \partial_{u}$, where $\operatorname{rank}\left(\xi^{i 1}, \xi^{i 2}, \eta^{i}\right)=2$. In the remainder of this section the parameter tuple $\theta=\left(\theta^{1}, \theta^{2}\right)$ runs through the set of pairs of smooth functions depending on $(x, u)$, and $i$ and $j$ run from 1 to 2 .

Definition 5. The module $\left\{Q^{\theta}\right\}$ is called singular for the differential function $L$ if for any $\theta$ with $\left(\theta^{i} \xi^{i 1}, \theta^{i} \xi^{i 2}\right) \neq(0,0)$ the vector field $Q^{\theta}$ is singular for $L$. The singularity co-order of the module $\left\{Q^{\theta}\right\}$ coincides with the maximum of the singularity co-orders of its elements.

By a point transformation, one of the basis vector fields, e.g. $Q^{2}$, can be reduced to $\partial_{u}$ (transforming $L$ simultaneously with $Q^{1}$ and $Q^{2}$.) Then $\left(\xi^{11}, \xi^{12}\right) \neq(0,0)$, and up to permutation of independent variables we can assume $\xi^{12} \neq 0$ and, therefore, set $\eta^{1}=0$ and $\xi^{12}=1$ by a change of basis. Any vector field from the module $\left\{Q^{\theta}\right\}$ with a nonzero value of $\theta^{1}$ is equivalent to the vector field $Q^{1}+\zeta Q^{2}$, where $\zeta=\theta^{2} / \theta^{1}$. All the other vector fields from $\left\{Q^{\theta}\right\}$ (which have $\theta^{1}=0$ and, therefore, are equivalent to $\partial_{u}$ ) can be neglected since each of them leads to the equation $\theta^{2}(x, u)=0$ which completely determines $u$ and therefore, does not give an ansatz for $u$.

This justifies why, up to point transformations, it suffices to study only singular sets of vector fields of the form $\left\{Q^{\zeta}=\xi \partial_{1}+\partial_{2}+\zeta \partial_{u}\right\}$, with $\xi$ a fixed smooth function of $(x, u)$ and $\zeta$ running through all such functions. The latter form of singular sets of vector fields will be called reduced.

Further simplification depends on whether the module is closed under the Lie bracket. In case it is, it can be assumed to be generated by two commuting vector fields which can be 
simultaneously reduced by a point transformation to shift operators, e.g., $Q^{1}=\partial_{2}$ and $Q^{2}=\partial_{u}$. Thus in the reduced form $\xi$ can be put to 0 . If the module is not closed under the Lie bracket, we have $\xi_{u} \neq 0$ in the reduced form. After the point transformation $\tilde{x}_{i}=x_{i}$ and $\tilde{u}=\xi$ and a change of basis, we obtain the basis $\tilde{Q}^{1}=\tilde{u} \partial_{\tilde{1}}+\partial_{\tilde{2}}$ and $\tilde{Q}^{2}=\partial_{\tilde{u}}$. Hence:

Proposition 1. In any two-dimensional module of vector fields in the space of three variables $\left(x_{1}, x_{2}, u\right)$, any basis vector fields $Q^{1}$ and $Q^{2}$ can be locally reduced, by point transformations, to the form $Q^{1}=\partial_{2}$ (resp. $Q^{1}=u \partial_{1}+\partial_{2}$ ) and $Q^{2}=\partial_{u}$ if the module is closed (resp. not closed) with respect to the Lie bracket of vector fields.

Theorem 1. A differential function $L$ with one dependent and two independent variables possesses a kth co-order singular two-dimensional module of vector fields if and only if it can be represented, up to point transformations, in the form

$$
L=\check{L}\left(x, \Omega_{r, k}\right),
$$

where $\Omega_{r, k}=\left(\omega_{\alpha}=D_{1}^{\alpha_{1}}\left(\xi D_{1}+D_{2}\right)^{\alpha_{2}} u, \alpha_{1} \leqslant k, \alpha_{1}+\alpha_{2} \leqslant r\right), \xi \in\{0, u\}$, and $\check{L}_{\omega_{\alpha}} \neq 0$ for some $\omega_{\alpha}$ with $\alpha_{1}=k$.

Proof. Suppose that a differential function $L$ possesses a $k$ th co-order singular two-dimensional module of vector fields $\left\{Q^{\theta}=\theta^{i} Q^{i}\right\}$. By a point transformation and a change of basis, we represent the basis elements in the reduced form $Q^{1}=\xi \partial_{1}+\partial_{2}$ and $Q^{2}=\partial_{u}$, where $\xi \in\{0, u\}$, and choose the subset $\left\{Q^{\zeta}=\xi \partial_{1}+\partial_{2}+\zeta \partial_{u}\right\}$ in $\left\{Q^{\theta}\right\}$, where $\zeta$ runs through the set of smooth functions of $(x, u)$. The initial differential function also will be changed by these transformations but throughout we will use the old notations for all new values.

We fix an arbitrary point $z^{0}=\left(x^{0}, u_{(r)}^{0}\right) \in J^{r}$ and consider the vector fields from $\left\{Q^{\zeta}\right\}$ for which $z^{0} \in \mathcal{Q}_{(r)}^{\zeta}$. This condition implies that the values of the derivatives of $\zeta$ with respect to only $x_{1}$ and $x_{2}$ in the point $\left(x^{0}, u^{0}\right)$ are expressed via $u_{(r)}^{0}$ and values of derivatives of $\zeta$ in $\left(x^{0}, u^{0}\right)$, containing differentiation with respect to $u$. The latter values are not constrained.

We introduce the new coordinates $\left\{x_{i}, \omega_{\alpha}=D_{1}^{\alpha_{1}}\left(\xi D_{1}+D_{2}\right)^{\alpha_{2}} u,|\alpha| \leqslant r\right\}$ in $J^{r}$ instead of the standard ones $\left\{x_{i}, u_{\alpha},|\alpha| \leqslant r\right\}$. This is a valid change of coordinates since the Jacobian matrix $\left(\partial \omega_{\alpha} / \partial u_{\alpha^{\prime}}\right)$ is nondegenerate. Indeed, it is a triangular matrix with all diagonal entries equal to 1 if the following order of multi-indices is implemented: $\alpha<\beta: \Leftrightarrow|\alpha|<|\beta| \vee\left(|\alpha|=|\beta| \wedge \alpha_{2}<\beta_{2}\right)$. Note that $\omega_{\alpha}=D_{1}^{\alpha_{1}}\left(\xi D_{1}+D_{2}\right)^{\alpha_{2}} u=D_{1}^{\alpha_{1}}\left(Q^{\zeta}\right)^{\alpha_{2}} u$ on $\mathcal{Q}_{(r)}^{\zeta}$.

Consider the differential function $\hat{L}$ obtained from $L$ by the above procedure of excluding, on the manifold $\mathcal{Q}_{(r)}^{\zeta}$, the derivatives of $u$ involving differentiations with respect to $x_{2}$ (see (1)). Since $Q^{\zeta}$ is a $k$ th co-order singular vector field for $L$, the function $\hat{L}$ does not depend on the derivatives $u_{(\kappa, 0)}, \kappa=k+1, \ldots, r$. We use this condition step-by-step, starting from the greatest value of $\kappa$ and re-writing the derivatives in the new coordinates of $J^{r}$ and in terms of $L$.

Thus, in the new coordinates the equation $\hat{L}_{u_{(r, 0)}}\left(z^{0}\right)=0$ has the form $L_{\omega_{(r, 0)}}\left(z^{0}\right)=0$. This completes the first step. Then in the second step the equation $\hat{L}_{u_{(r-1,0)}}\left(z^{0}\right)=0$ implies that

$$
L_{\omega_{(r-1,0)}}\left(z^{0}\right)+L_{\omega_{(r-1,1)}}\left(z^{0}\right) \zeta_{u}\left(x^{0}, u^{0}\right)=0 .
$$

We split with respect to the value $\zeta_{u}\left(x^{0}, u^{0}\right)$ since it is unconstrained. As a result, we obtain the equations $L_{\omega_{(r-1,0)}}\left(z^{0}\right)=0$ and $L_{\omega_{(r-1,1)}}\left(z^{0}\right)=0$.

Iterating this procedure, before the $\mu$ th step, $\mu \in\{1, \ldots, r-k\}$, we derive the equations $L_{\omega_{\left(r-\mu^{\prime}, \nu\right)}}\left(z^{0}\right)=0, \mu^{\prime}=0, \ldots, \mu-2, \nu=0, \ldots, \mu^{\prime}$. Then the equation $\hat{L}_{u_{(r-\mu+1,0)}}\left(z^{0}\right)=0$ implies that

$$
\left.\sum_{\nu=0}^{\mu-1} L_{\omega_{(r-\mu+1, \nu)}}\left(z^{0}\right)\left(\partial_{u}\left(Q^{\zeta}\right)^{\nu} u\right)\right|_{(x, u)=\left(x^{0}, u^{0}\right)}=0 .
$$


The values $\partial_{u}^{\nu+1} \zeta\left(x^{0}, u^{0}\right), \nu=0, \ldots, \mu-1$, are unconstrained. Splitting with respect to them, which is equivalent to splitting with respect to $\left.\left(\partial_{u}\left(Q^{\zeta}\right)^{\nu} u\right)\right|_{(x, u)=\left(x^{0}, u^{0}\right)}, \nu=0, \ldots, \mu-1$, gives the equations $L_{\omega_{(r-\mu+1, \nu)}}\left(z^{0}\right)=0, \nu=0, \ldots, \mu-1$.

Finally, after the $(r-k)$ th step we derive the system $L_{\omega_{\left(r-\mu^{\prime}, \nu\right)}}\left(z^{0}\right)=0, \mu^{\prime}=0, \ldots, r-k+1$, $\nu=0, \ldots, \mu^{\prime}$, which implies condition (2).

Conversely, let an $r$ th order differential function $L$ be of the form (2) (after a point transformation). For an arbitrary smooth function $\zeta=\zeta(x, u)$ we consider the vector field $Q^{\zeta}=\xi \partial_{1}+\partial_{2}+\zeta \partial_{u}$ and the differential function $\tilde{L}=\check{L}\left(x, \tilde{\Omega}_{r, k}\right)$ where

$$
\tilde{\Omega}_{r, k}=\left(\omega_{\alpha}=D_{1}^{\alpha_{1}}\left(Q^{\zeta}\right)^{\alpha_{2}} u, \alpha_{1} \leqslant k, \alpha_{1}+\alpha_{2} \leqslant r\right) .
$$

Then ord $\tilde{L}=k$ and

$$
\left.L\right|_{\mathcal{Q}_{(r)}^{\zeta}}=\left.\tilde{L}\right|_{\mathcal{Q}_{(r)}^{\zeta}}
$$

i.e., $\left\{Q^{\zeta}=Q^{1}+\zeta Q^{2}\right\}$, where $Q^{1}=\xi \partial_{1}+\partial_{2}, Q^{2}=\partial_{u}$ and $\zeta$ runs through the set of smooth functions of $(x, u)$, is a $k$ th co-order singular set for the differential function $L$ in the new variables. We complete the set by the vector fields equivalent to its elements or $\partial_{u}$ and return to the old variables. As a result, for the differential function $L$ we construct a $k$ th co-order singular two-dimensional module of vector fields $\left\{Q^{\theta}=\theta^{i} Q^{i}\right\}$.

Corollary 2. A differential function with one dependent and two independent variables admits a kth co-order singular two-dimensional module generated by commuting vector fields if and only if it can be reduced by a point transformation of the variables to a differential function in which all differentiations with respect to one of the independent variables are of order $\leq k$.

Corollary 3. Any differential function with one dependent and two independent variables (not identically vanishing) admits no ultra-singular two-dimensional module of singular vector fields.

Note 1. It is obvious that a singular module may contain vector fields whose singularity coorders are less than the singularity co-order of the whole module. Suppose that $\left\{Q^{\zeta}=\xi \partial_{1}+\right.$ $\left.\partial_{2}+\zeta \partial_{u}\right\}$ is a singular set of vector fields for a differential function $L$, and its singularity co-order equals $k$. Then the values of $\zeta$ for which $\operatorname{sco}_{L} Q^{\zeta}<k$ are solutions of the equation

$$
\sum_{\nu=0}^{r-k} \check{L}_{\omega_{(k, \nu)}}\left(x, \tilde{\Omega}_{r, k}\right)\left(\partial_{u}\left(Q^{\zeta}\right)^{\nu} u\right)=0,
$$

where $\tilde{\Omega}_{r, k}=\left(D_{1}^{\alpha_{1}}\left(Q^{\zeta}\right)^{\alpha_{2}} u, \alpha_{1} \leqslant k, \alpha_{1}+\alpha_{2} \leqslant r\right)$ and $\check{L}$ is defined in Theorem 1 . In other words, the regular values of $\zeta$ associated with the vector fields of the maximal singularity co-order $k$ in $\left\{Q^{\zeta}\right\}$ satisfy the inequality

$$
\sum_{\nu=0}^{r-k} \check{L}_{\omega_{(k, \nu)}}\left(x, \tilde{\Omega}_{r, k}\right)\left(\partial_{u}\left(Q^{\zeta}\right)^{\nu} u\right) \neq 0 .
$$

\section{Singular vector fields of differential equations}

We will say that a vector field $Q$ is (strongly) singular for a differential equation $\mathcal{L}$ if it is singular for the differential function $L[u]$ which is the left hand side of the canonical representation $L[u]=0$ of the equation $\mathcal{L}$. Usually we will omit the attribute "strongly".

Since left hand sides of differential equations are defined up to multipliers which are nonvanishing differential functions, the conditions from Definition 4 can be weakened when considering differential equations. 
Definition 6. A vector field $Q$ is called weakly singular for the differential equation $\mathcal{L}: L[u]=0$ if there exist a differential function $\tilde{L}=\tilde{L}[u]$ of an order less than $r$ and a nonvanishing differential function $\lambda=\lambda[u]$ of an order not greater than $r$ such that $\left.L\right|_{\mathcal{Q}_{(r)}}=\left.\lambda \tilde{L}\right|_{\mathcal{Q}_{(r)}}$. Otherwise $Q$ is called a weakly regular vector field for the differential equation $\mathcal{L}$. If the minimal order of differential functions whose restrictions on $\mathcal{Q}_{(r)}$ coincide, up to nonvanishing functional multipliers, with $\left.L\right|_{\mathcal{Q}_{(r)}}$ is equal to $k(k<r)$ then the vector field $Q$ is said to be weakly singular of co-order $k$ for the differential equation $\mathcal{L}$.

The notions of ultra-singularity in the weak and the strong sense coincide. Analogous to the case of strong regularity, weakly regular vector fields for the differential equation $\mathcal{L}$ are defined to have weak singularity co-order $r=$ ord $L$. The weak singularity co-order of a vector field $Q$ for an equation $\mathcal{L}$ will be denoted by $\operatorname{wsco}_{\mathcal{L}} Q$.

Note that strong singularity implies weak singularity and hence weak regularity implies strong regularity. It is obvious that the weak singularity co-order is never greater and may be less than the strong singularity co-order. In particular, strongly regular vector fields may be singular in the weak sense. For example, the equation $u_{t t t}=e^{u_{x x}}\left(u_{x}+u\right)$ possesses the singular vector field $\partial_{t}$ whose strong and weak singularity co-order equal 2 and 1 , respectively. The same vector field $\partial_{t}$ is strongly regular and is of weak singularity co-order 1 for the equation $u_{t}=e^{u_{x x}}\left(u_{x}+u\right)$.

If $Q$ is a weakly singular vector field for $\mathcal{L}$ then any vector field equivalent to $Q$ is weakly singular for $\mathcal{L}$ with the same co-order of weak singularity.

Weakly singular vector fields are related to characteristic directions (cf. [14] concerning characteristic directions and characteristic hypersurfaces): Given a vector field $Q=\xi^{i}(x, u) \partial_{i}+$ $\eta(x, u) \partial_{u}$ weakly singular for a differential equation $\mathcal{L}$, in each point of the manifold $\mathcal{L}$ the vector $\left(\xi^{1}, \xi^{2}\right)$ is orthogonal to a characteristic direction of the equation $\mathcal{L}$ in this point.

Let $\hat{L}$ be a differential function associated with $L$ on the manifold $\mathcal{Q}_{(r)}$, namely, obtained from $L$ via excluding those derivatives of $u$ which contain differentiations with respect to $x_{2}$ in view of equations defining $\mathcal{Q}_{(r)}$. Suppose additionally that $\hat{L}$ is of maximal rank in the derivative $u$ of the highest order $k$ appearing in this differential function, i.e., $\hat{L}_{u_{(k, 0)}} \neq 0$ on the solution manifold of the equation $\hat{L}=0$. Then the weak singularity co-order of $Q$ for the equation $\mathcal{L}: L=0$ equals the order $k$ of $\hat{L}$ and, therefore, the strong singularity co-order of $Q$. Hence in this case testing that a vector field is weakly singular for a partial differential equation with two independent variables can be implemented via an entirely algorithmic procedure.

Theorem 2. An rth order differential equation $\mathcal{L}: L[u]=0$ of maximal rank with one dependent and two independent variables possesses a kth co-order weakly singular two-dimensional module of vector fields if and only if $L$ can be represented, up to point transformations, in the form

$$
L=\Lambda[u] \check{L}\left(x, \Omega_{r, k}\right)
$$

where $\Lambda$ is a nonvanishing differential function of order not greater than $r, \Omega_{r, k}=\left(\omega_{\alpha}=\right.$ $\left.D_{1}^{\alpha_{1}}\left(\xi D_{1}+D_{2}\right)^{\alpha_{2}} u, \alpha_{1} \leqslant k, \alpha_{1}+\alpha_{2} \leqslant r\right), \xi \in\{0, u\}$, and $\check{L}_{\omega_{\alpha}} \neq 0$ for some $\omega_{\alpha}$ with $\alpha_{1}=k$.

Proof. We will freely use the notations and definitions from the proof of Theorem 1,

Suppose at first that a differential equation $\mathcal{L}: L[u]=0$ is of maximal rank and admits a $k$ th co-order weakly singular two-dimensional module of vector fields. Up to point transformations and changes of module basis, we may consider only a set $\left\{Q^{\zeta}=\xi \partial_{1}+\partial_{2}+\zeta \partial_{u}\right\}$ of singular vector fields in reduced form.

We fix an arbitrary point $z^{0}=\left(x^{0}, u_{(r)}^{0}\right) \in \mathcal{L} \subset J^{r}$ and choose the vector fields from $\left\{Q^{\zeta}\right\}$ for which $z^{0} \in \mathcal{Q}_{(r)}^{\zeta}$. This condition implies that the values of derivatives of $\zeta$ with respect to only $x_{1}$ and $x_{2}$ in the point $\left(x^{0}, u^{0}\right)$ are expressed via $u_{(r)}^{0}$ and values of derivatives of $\zeta$ in $\left(x^{0}, u^{0}\right)$, containing differentiation with respect to $u$. The latter values are not constrained. 
The differential function $\hat{L}$ is obtained from $L$ by excluding, on the manifold $\mathcal{Q}_{(r)}^{\zeta}$, derivatives of $u$ involving differentiations with respect to $x_{2}$ (see (10). $k$ th co-order weak singularity of $Q^{\zeta}$ for $L$ leads to $\hat{L}_{u_{(\kappa, 0)}}\left(z_{0}\right)=0, \kappa=k+1, \ldots, r$. We use this condition step-by-step as in the proof of Theorem 1, starting from the greatest value of $\kappa$ and re-writing the derivatives in the new coordinates $\left\{x_{i}, \omega_{\alpha}=D_{1}^{\alpha_{1}}\left(\xi D_{1}+D_{2}\right)^{\alpha_{2}} u,|\alpha| \leqslant r\right\}$ of $J^{r}$ and in terms of $L$. Therefore,

$$
L_{\omega_{\left(r-\mu^{\prime}, \nu\right)}}\left(z^{0}\right)=0, \quad \mu^{\prime}=0, \ldots, r-k+1, \quad \nu=0, \ldots, \mu^{\prime},
$$

which is satisfied for any $z^{0} \in \mathcal{L}$. Applying the Hadamard lemma to each of these equations and then simultaneously integrating them, we obtain (3) (cf. the proof of Theorem 1 in [26]).

Conversely, let an $r$ th order differential function $L$ be of the form (3) (after a point transformation). For an arbitrary smooth function $\zeta=\zeta(x, u)$ we consider the vector field $Q^{\zeta}=\xi \partial_{1}+\partial_{2}+\zeta \partial_{u}$ and the differential function $\tilde{L}=\check{L}\left(x, \tilde{\Omega}_{r, k}\right)$, where

$$
\tilde{\Omega}_{r, k}=\left(\omega_{\alpha}=D_{1}^{\alpha_{1}}\left(Q^{\zeta}\right)^{\alpha_{2}} u, \alpha_{1} \leqslant k, \alpha_{1}+\alpha_{2} \leqslant r\right) .
$$

Then ord $\tilde{L}=k$ and $\left.L\right|_{\mathcal{Q}_{(r)}^{\zeta}}=\left.\Lambda \tilde{L}\right|_{\mathcal{Q}_{(r)}^{\zeta}}$, i.e., $\left\{Q^{\zeta}=Q^{1}+\zeta Q^{2}\right\}$, where $Q^{1}=\xi \partial_{1}+\partial_{2}, Q^{2}=\partial_{u}$ and $\zeta$ runs through the set of smooth functions of $(x, u)$, is a $k$ th co-order weakly singular set for the differential equation $\mathcal{L}$ in the new variables. We complete the set by the vector fields equivalent to its elements or $\partial_{u}$ and return to the old variables, thereby constructing a $k$ th co-order weakly singular two-dimensional module of vector fields $\left\{Q^{\theta}=\theta^{i} Q^{i}\right\}$ for the differential equation $\mathcal{L}$.

Corollary 4. A differential equation $\mathcal{L}: L[u]=0$ of maximal rank with one dependent and two independent variables possesses a $k$ th co-order weakly singular two-dimensional module of vector fields if and only if this module is $k$ th co-order strongly singular for $\mathcal{L}$ (possibly in a representation differing from $L[u]=0$ in multiplication by a nonvanishing differential function of $u$ ).

Definition 7. A vector field $Q$ is called a singular reduction operator of a differential equation $\mathcal{L}$ if $Q$ is both a reduction operator of $\mathcal{L}$ and a weakly singular vector field of $\mathcal{L}$.

\section{Example: evolution equations}

In this section we investigate singular reduction operators of $(1+1)$-dimensional evolution equations of the form

$$
u_{t}=H\left(t, x, u_{(r, x)}\right) \text {, }
$$

where $r>1, u_{0}:=u, u_{k}=\partial^{k} u / \partial x^{k}, u_{(r, x)}=\left(u_{0}, u_{1}, \ldots, u_{r}\right)$ and $H_{u_{r}} \neq 0$. (We revert to the notation $t$ and $x$ for $x_{1}$ and $x_{2}$, respectively, and change the notations of the corresponding derivatives.) Evolution equations are quite specific from the point of view of singular vector fields and singular reduction operators.

Proposition 2. A vector field $Q=\tau(t, x, u) \partial_{t}+\xi(t, x, u) \partial_{x}+\eta(t, x, u) \partial_{u}$ is singular for the differential function $L=u_{t}-H\left(t, x, u_{(r, x)}\right)$ of order $r>1$ if and only if $\tau=0$. The co-order of singularity of any singular vector field for any such differential function equals 1.

Proof. Suppose that $\tau \neq 0$. Excluding the derivative $u_{t}$ from $L$ according to the equation $u_{t}=\eta / \tau-\xi u_{x} / \tau$ results in a differential function $\tilde{L}=\eta / \tau-\xi u_{x} / \tau-H\left(t, x, u_{(r, x)}\right)$. Since ord $\tilde{L}=r=\operatorname{ord} L$, the vector field $Q$ is not singular in this case. Therefore, for the vector field $Q$ to be singular, the coefficient $\tau$ has to vanish.

If $\tau=0$ and therefore $\xi \neq 0$, all the derivatives $u_{k}, k=1, \ldots, r$, can be expressed, on the manifold $\mathcal{Q}_{(r)}$ via $t, x$ and $u: u_{k}=\left(\partial_{x}+\zeta \partial_{u}\right)^{k-1} \zeta, k=1, \ldots, r$, where $\zeta=\eta / \xi$. Using these 
expressions for excluding the derivatives $u_{k}, k=1, \ldots, r$ from $L$, we obtain the differential function

$$
\tilde{L}=u_{t}-\tilde{H}(t, x, u), \quad \tilde{H}:=H\left(t, x, u, \zeta, \zeta_{x}+\zeta \zeta_{u}, \ldots,\left(\partial_{x}+\zeta \partial_{u}\right)^{r-1} \zeta\right),
$$

whose order equals 1 . Hence the vector field $Q$ is singular for the differential function $L$, and its singularity co-order equals 1.

Corollary 5. For any $(1+1)$-dimensional evolution equation, the corresponding differential function possesses exactly one set of singular vector fields in the reduced form, namely, $S=$ $\left\{\partial_{x}+\zeta(x, u) \partial_{u}\right\}$. The singularity co-order of $S$ equals 1 .

It is obvious that under the condition $H_{u_{r}} \neq 0$ a vector field is singular for the differential function $u_{t}-H\left(t, x, u_{(r, x)}\right)$ if and only if it is weakly singular for the differential equation $u_{t}=H\left(t, x, u_{(r, x)}\right)$. Hence we do not distinguish between strong and weak singularity (cf. Corollary (4).

The vector fields $\partial_{2}$ and $\partial_{u}$ generating the singular module associated with $S$ commute and the differential function $L$ contains only first order differentiation with respect to $t$ (namely, in the form of the derivative $u_{t}$ ). This perfectly agrees with Corollary 2 ,

We fix an arbitrary equation $\mathcal{L}$ of the form (4) and denote by $\mathcal{Q}_{0}(\mathcal{L})$ the set of reduction operators of $\mathcal{L}$, belonging to $S$. For the equation $\mathcal{L}$ and $Q \in \mathcal{Q}_{0}(\mathcal{L})$, the conditional invariance criterion implies only the single $r$ th order equation

$$
\zeta_{t}+\zeta_{u} \tilde{H}=\tilde{H}_{x}+\zeta \tilde{H}_{u}, \quad \tilde{H}:=H\left(t, x, u, \zeta, \zeta_{x}+\zeta \zeta_{u}, \ldots,\left(\partial_{x}+\zeta \partial_{u}\right)^{r-1} \zeta\right),
$$

with respect to the single unknown function $\zeta$ with three independent variables $t, x$ and $u$, which we will denote by $\mathrm{DE}_{0}(\mathcal{L})$. In other words, the system of determining equations in this case consists of the single equation $\mathrm{DE}_{0}(\mathcal{L})$ and, therefore, is not overdetermined. $\mathrm{DE}_{0}(\mathcal{L})$ is the compatibility condition of the equations $u_{x}=\zeta$ and $\mathcal{L}$.

Theorem 3. Up to the equivalences of operators and solution families, for any equation of form (4) there exists a one-to-one correspondence between one-parametric families of its solutions and reduction operators with zero coefficients of $\partial_{t}$. Namely, each operator of this kind corresponds to the family of solutions which are invariant with respect to this operator. The problems of the construction of all one-parametric solution families of equation (4) and the exhaustive description of its reduction operators with zero coefficients of $\partial_{t}$ are completely equivalent.

Proof. Let $\mathcal{L}$ be an equation from class (4) and $Q=\partial_{x}+\zeta \partial_{u} \in \mathcal{Q}_{0}(\mathcal{L})$, i.e., the coefficient $\zeta=\zeta(t, x, u)$ satisfies the equation $\operatorname{DE}_{0}(\mathcal{L})$. An ansatz constructed with $Q$ has the form $u=$ $f(t, x, \varphi(\omega))$, where $f=f(t, x, \varphi)$ is a given function, $f_{\varphi} \neq 0, \varphi=\varphi(\omega)$ is the new unknown function and $\omega=t$ is the invariant independent variable. This ansatz reduces $\mathcal{L}$ to a firstorder ordinary differential equation $\mathcal{L}^{\prime}$ in $\varphi$, solvable with respect to $\varphi^{\prime}$. The general solution of the reduced equation $\mathcal{L}^{\prime}$ can be represented in the form $\varphi=\varphi(\omega, \varkappa)$, where $\varphi_{\varkappa} \neq 0$ and $\varkappa$ is an arbitrary constant. Substituting this solution into the ansatz results in the one-parametric family $\mathcal{F}$ of solutions $u=\tilde{f}(t, x, \varkappa)$ of $\mathcal{L}$ with $\tilde{f}=f(t, x, \varphi(t, \varkappa))$. Expressing the parameter $\varkappa$ from the equality $u=\tilde{f}(t, x, \varkappa)$, we obtain that $\varkappa=\Phi(t, x, u)$, where $\Phi_{u} \neq 0$. Then $\zeta=u_{x}=$ $-\Phi_{x} / \Phi_{u}$ for any $u \in \mathcal{F}$, i.e., for any admissible value of $(t, x, \varkappa)$. This implies that $\zeta=-\Phi_{x} / \Phi_{u}$ for any admissible value of $(t, x, u)$.

Conversely, suppose that $\mathcal{F}=\{u=f(t, x, \varkappa)\}$ is a one-parametric family of solutions of $\mathcal{L}$. The derivative $f_{\varkappa}$ is nonzero since the parameter $\varkappa$ is essential. We express $\varkappa$ from the equality $u=f(t, x, \varkappa): \varkappa=\Phi(t, x, u)$ for some function $\Phi=\Phi(t, x, u)$ with $\Phi_{u} \neq 0$. Consider the operator $Q=\partial_{x}+\zeta \partial_{u}$, where the coefficient $\zeta=\zeta(t, x, u)$ is defined by $\zeta=-\Phi_{x} / \Phi_{u} . Q[u]=0$ for any $u \in \mathcal{F}$. The ansatz $u=f(t, x, \varphi(\omega))$, where $\omega=t$, associated with $Q$, reduces $\mathcal{L}$ to the equation $\varphi_{\omega}=0$. Therefore [26], $Q \in \mathcal{Q}_{0}(\mathcal{L})$ and hence the function $\zeta$ satisfies $\operatorname{DE}_{0}(\mathcal{L})$. 
Corollary 6. The nonlinear $(1+2)$-dimensional evolution equation $\mathrm{DE}_{0}(\mathcal{L})$ is reduced by the composition of the nonlocal substitution $\zeta=-\Phi_{x} / \Phi_{u}$, where $\Phi$ is a function of $(t, x, u)$, and the hodograph transformation

$\begin{array}{ll}\text { the new independent variables: } & \tilde{t}=t, \quad \tilde{x}=x, \quad \varkappa=\Phi, \\ \text { the new dependent variable: } & \tilde{u}=u\end{array}$

to the initial equation $\mathcal{L}$ in the function $\tilde{u}=\tilde{u}(\tilde{t}, \tilde{x}, \varkappa)$ with $\varkappa$ playing the role of a parameter.

Note 2. One-parametric families $u=f(t, x, \varkappa)$ and $u=\tilde{f}(t, x, \tilde{\varkappa})$ are defined to be equivalent if they consist of the same functions and differ only by parameterizations, i.e., if there exists a function $\zeta=\zeta(\varkappa)$ such that $\zeta_{\varkappa} \neq 0$ and $\tilde{f}(t, x, \zeta(\varkappa))=f(t, x, \varkappa)$. Equivalent one-parametric families of solutions are associated with the same operator from $\mathcal{Q}_{0}(\mathcal{L})$ and have to be identified.

Note 3. The triviality of the above ansatz and the reduced equation results from the above special representation for the solutions of the determining equation. Under this approach difficulties in the construction of ansatzes and the integration of the reduced equations are replaced by difficulties in obtaining the representation for the coefficients of the reduction operators.

The above consideration shows that for any evolution equation $\mathcal{L}$ the conventional partition of the set $\mathfrak{Q}(\mathcal{L})$ of its reduction operators with the conditions $\tau \neq 0$ and $\tau=0$ is natural since it coincides with the partition of $\mathfrak{Q}(\mathcal{L})$ into the singular and regular subsets. This is a specific property of evolution equations which does not hold for general partial differential equations in two independent variables. After factorizing the subsets of $\mathfrak{Q}(\mathcal{L})$ with respect to the usual equivalence relation of reduction operators, we obtain two different cases of inequivalent reduction operators (the regular case $\tau=1$ and the singular case $\tau=0$ and $\xi=1$ ), which have to be studied separately.

Singular reduction operators of $\mathcal{L}$ are described in a unified 'no-go' way. All singular reduction operators of $\mathcal{L}$ have the same singularity co-order equal to 1 and hence reduce $\mathcal{L}$ to first order ordinary differential equations. The coincidence of the singularity co-orders guarantees the existence of a bijection between the set of singular reduction operators of $\mathcal{L}$ and the set of oneparametric families of its solutions (up to the natural equivalence relations in these sets). As a result, in the case $\tau=0$ and $\xi=1$ the determining equation for a single coefficient of $\partial_{u}$ is reduced, with no additional assumptions and conditions, to the initial equation $\mathcal{L}$ by a nonlocal transformation (cf. Corollary 6).

The regular case $\tau=1$ is more complicated than the singular one. It essentially depends on the structure of the equation including the order, the kind of nonlinearities, etc. Up to now there are no exhaustive results on regular reduction operators even for second-order evolution equations. Only certain subclasses of such equations were investigated. See, e.g., [1, 5, 21, 22] for the complete classifications of regular reduction operators for some subclasses of second-order evolution equations parameterized by functions of single arguments. For example, even for the class of nonlinear diffusion equations of the general form $u_{t}=\left(f(u) u_{x}\right)_{x}$ (a classical example of solving a group classification problem for partial differential equations [17]), the set of values of the parameter-function $f$ which correspond to equations possessing non-Lie regular reduction operators has not yet been found. Most evolution equations have no regular reduction operators. A simple example is

$$
u_{t}=u_{x x}+u e^{u_{x}}+x e^{2 u_{x}}+t e^{3 u_{x}}+e^{4 u_{x}}+e^{5 u_{x}} .
$$

Some evolution equations (the linear ones [10, 21], Burgers' equation [13], etc.) possess so many regular reduction operators that 'no-go' statements like those for singular reduction operators are true for them, but the nature of this 'no-go' differs from the 'no-go' of the singular case and is related to the property of linearity or linearizability of the corresponding evolution equations. 


\section{Example: nonlinear wave equations}

The next example which we study in detail within the framework of singular reduction operators is given by the class of nonlinear wave equations (in the characteristic, or light-cone, variables) of the general form

$$
u_{12}=F(u) .
$$

Here $F$ is an arbitrary smooth function of $u$. This class essentially differs from the class of evolution equations within the framework of singular vector fields. The main differences are that each differential function corresponding to an equation from class (5) has two singular sets of vector fields and these sets contain vector fields of lower singularity co-orders than the singularity co-orders of the whole sets. Thus, for any $F$ the vector field $Q=\xi^{i}(x, u) \partial_{i}+\eta(x, u) \partial_{u}$ is singular for the corresponding differential function $L=u_{12}-F(u)$ if and only if $\xi^{1} \xi^{2}=0$. Moreover, it is obvious that there are no differences between strong and weak singularity of vector fields for equations from the class (5). Indeed, suppose that $\xi^{2} \neq 0$. Excluding the derivatives $u_{2}$ and $u_{12}$ from $L$ according to (1), we obtain a differential function $\tilde{L}$ with the coefficient $-\xi^{1} / \xi^{2}$ of $u_{11}$. We have ord $\tilde{L}<2$ if and only if $\xi^{1}=0$.

Therefore, for any $F$ the differential function $L=u_{12}-F(u)$ possesses exactly two sets of singular vector fields in the reduced form, $S=\left\{\partial_{2}+\zeta(x, u) \partial_{u}\right\}$ and $S^{*}=\left\{\partial_{1}+\zeta^{*}(x, u) \partial_{u}\right\}$. The vector fields equivalent to $\partial_{u}$ are not suitable as reduction operators. Any singular vector field of $L$ is equivalent to one of the above fields. Moreover, each equation of the form (5) admits the discrete symmetry transformation permuting the variables $x_{1}$ and $x_{2}$. This transformation generates a one-to-one mapping between $S$ and $S^{*}$ (cf. Corollary 1). Hence it suffices, up to equivalence of vector fields (and permutation of $x_{1}$ and $x_{2}$ ), to investigate only singular reduction operators from the set $S$.

For an equation $\mathcal{L}$ from class (5) and an operator $Q=\partial_{2}+\zeta \partial_{u}$ the conditional invariance criterion takes the form

$$
\left.\left(\zeta_{12}+\zeta_{1 u} u_{2}+\zeta_{2 u} u_{1}+\zeta_{u u} u_{1} u_{2}+\zeta_{u} u_{12}\right)\right|_{\mathcal{L} \cap \mathcal{Q}_{(2)}}=\zeta F_{u}
$$

The intersection $\mathcal{L} \cap \mathcal{Q}_{(2)}$ is singled out from $J^{2}$ by the equations $u_{2}=\zeta, \zeta_{1}+\zeta_{u} u_{1}=F$ and $u_{12}=F$. Our further considerations therefore depend on the values of $\zeta_{u}$ and $F_{u}$. We analyze all the possible cases.

Let $\zeta_{u}=0$ and $F_{u}=0$. Then $Q$ is an ultra-singular vector field for the differential function $L$. The third equation defining $\mathcal{L} \cap \mathcal{Q}_{(2)}$ takes the form $\zeta_{1}=F$ and contains no derivatives of $u$. It should be assumed as a condition with respect to $\zeta$ and hence the conditional invariance criterion is identically satisfied in this case. An ansatz constructed with the operator $Q$ is $u=\varphi(\omega)+\int \zeta d x_{2}$, where $\omega=x_{1}$. It reduces equation (5) to an identity. This is explained by the ultra-singularity of the reduction operator $Q$.

If $\zeta_{u}=0$ and $F_{u} \neq 0$, the singularity co-order of $Q$ for the differential function $L$ equals 0 . The third equation defining $\mathcal{L} \cap \mathcal{Q}_{(2)}$ again takes the form $\zeta_{1}=F$ but now can be solved with

respect to $u: u=\check{F}\left(\zeta_{1}\right)$, where $\check{F}$ is the inverse to $F$. Then the conditional invariance criterion is equivalent to the equation $\zeta_{12}=\zeta F_{u}\left(\check{F}\left(\zeta_{1}\right)\right)$ with respect to $\zeta$. The ansatz constructed with the operator $Q$ reduces equation (5) to the algebraic equation $F\left(\varphi+\int \zeta d x_{2}\right)=\zeta_{1}$ for the function $\varphi$ in agreement with the singularity co-order 0 of $Q$. Indeed, inverting $F$, we obtain the equality $\varphi=\check{F}\left(\zeta_{1}\right)-\int \zeta d x_{2}$ whose right-hand side does not depend on $x_{2}$ in view of the equation on $\zeta$. Conversely, let us fix a solution $u=f(x)$ of equation (5) and set $\zeta=f_{2}$. Then $\zeta_{12}=\zeta F_{u}\left(\check{F}\left(\zeta_{1}\right)\right)$, i.e., in view of the conditional invariance criterion $Q=\partial_{2}+\zeta \partial_{u}$ is a reduction operator of equation (5), and $\zeta_{u}=0$. The solution $u=f(x)$ is invariant with respect to $Q$. The above results can be summed up as follows: 
Theorem 4. For any equation from class (5) with $F_{u} \neq 0$ there exists a one-to-one correspondence between its solutions and reduction operators of the form $Q=\partial_{2}+\zeta(x) \partial_{u}$ (resp. $\left.Q^{*}=\partial_{1}+\zeta^{*}(x) \partial_{u}\right)$. Namely, each operator of this kind is of singularity co-order 0 and corresponds to the solution which is invariant with respect to this operator. The problems of solving an equation from class (5) with $F_{u} \neq 0$ and the exhaustive description of its reduction operators of the above form are completely equivalent.

Corollary 7. Any solution $u=f(x)$ of equation (5) with $F_{u} \neq 0$ is invariant with respect to two reduction operators $Q=\partial_{2}+\zeta(x) \partial_{u}$ and $Q^{*}=\partial_{1}+\zeta^{*}(x) \partial_{u}$ of equation (5), having singularity co-order 0 . Here $\zeta=f_{2}$ and $\zeta^{*}=f_{1}$. The property of possessing the same invariant solution of equation (5) establishes a canonical bijection $Q \leftrightarrow Q^{*}$ between the sets of reduction operators of singularity co-order 0 . The adjoint values of $\zeta$ and $\zeta^{*}$ are connected by the formulas

$$
\zeta^{*}=\frac{\zeta_{11}}{F_{u}\left(\check{F}\left(\zeta_{1}\right)\right)}, \quad \zeta=\frac{\zeta_{22}^{*}}{F_{u}\left(\check{F}\left(\zeta_{2}^{*}\right)\right)} .
$$

The regular values of $\zeta$ for which the singularity co-order of $Q$ coincides with the singularity co-order of the whole family $S$ (and equals 1 ) satisfy the condition $\zeta_{u} \neq 0$. The third equation defining $\mathcal{L} \cap \mathcal{Q}_{(2)}$ then provides the following expression for $u_{1}$ :

$$
u_{1}=\frac{F-\zeta_{1}}{\zeta_{u}}=: \zeta^{*}
$$

The conditional invariance criterion implies only the single equation

$$
\zeta_{12}+\zeta \zeta_{1 u}+\left(\zeta_{2 u}+\zeta \zeta_{u u}\right) \frac{F-\zeta_{1}}{\zeta_{u}}+\zeta_{u} F=\zeta F_{u}
$$

with respect to the single function $\zeta$, i.e., in this case the system of determining equations consists of the single equation (6) and, therefore, is not overdetermined.

Equation (6) can be rewritten in the form of the compatibility condition

$$
\zeta_{1}+\zeta^{*} \zeta_{u}=\zeta_{2}^{*}+\zeta \zeta_{u}^{*}=F
$$

of the equations $u_{1}=\zeta^{*}, u_{2}=\zeta$ and $u_{12}=F$. It is obvious that $\zeta_{u}^{*} \neq 0$. Due to symmetry with respect to the permutation of $x_{1}$ and $x_{2}$, we obtain the following statement.

Proposition 3. For any equation from class (5), there exists a canonical bijection $Q \leftrightarrow Q^{*}$ between sets of its singular reduction operators of the forms $Q=\partial_{2}+\zeta(x, u) \partial_{u}$ and $Q^{*}=$ $\partial_{1}+\zeta^{*}(x, u) \partial_{u}$, where $\zeta_{u} \neq 0$ and $\zeta_{u}^{*} \neq 0$. This bijection is given by the formulas

$$
Q \rightarrow Q^{*}: \quad \zeta^{*}=\frac{F-\zeta_{1}}{\zeta_{u}}, \quad Q^{*} \rightarrow Q: \quad \zeta=\frac{F-\zeta_{2}^{*}}{\zeta_{u}^{*}}
$$

A solution of equation (5) is invariant with respect to the operator $Q$ if and only if it is invariant with respect to the operator $Q^{*}$.

Theorem 5. Up to the equivalence of solution families, for any equation from class (5) with $F_{u} \neq 0$ there exists a one-to-one correspondence between one-parametric families of its solutions and reduction operators of the form $Q=\partial_{2}+\zeta(x, u) \partial_{u}$, where $\zeta_{u} \neq 0$ (resp. $Q^{*}=\partial_{1}+\zeta^{*}(x, u) \partial_{u}$, where $\left.\zeta_{u}^{*} \neq 0\right)$. Namely, any such operator corresponds to the family of solutions which are invariant with respect to this operator. The problems of the construction of all one-parametric solution families of an equation from class (5) with $F_{u} \neq 0$ and the exhaustive description of its reduction operators of the above form are completely equivalent. 
Proof. In view of Proposition 3 , it is sufficient to consider only operators with zero coefficient of $\partial_{1}$. Although the proof is similar to the proof of the analogous statement for evolution equations it differs from it in essential details and will therefore be presented completely.

An ansatz constructed with the operator $Q=\partial_{2}+\zeta(x, u) \partial_{u}$ has the form $u=f(x, \varphi(\omega))$, where $f=f(x, \varphi)$ is a given function, $f_{\varphi} \neq 0, \varphi=\varphi(\omega)$ is the new unknown function and $\omega=x_{1}$ is the invariant independent variable. Here $\zeta_{u} \neq 0$ implies $f_{2 \varphi} \neq 0$. Hence this ansatz reduces equation (5) to a first-order ordinary differential equation $\mathcal{L}^{\prime}$ in $\varphi$, which is solvable with respect to $\varphi^{\prime}$. The general solution of the reduced equation $\mathcal{L}^{\prime}$ essentially depends on an arbitrary constant $\varkappa: \varphi=\varphi(\omega, \varkappa)$, where $\varphi_{\varkappa} \neq 0$. Substituting the general solution into the ansatz gives the one-parametric family $\mathcal{F}$ of solutions $u=\tilde{f}(x, \varkappa)$ of (5) with $\tilde{f}=f\left(x, \varphi\left(x_{1}, \varkappa\right)\right)$.

Conversely, suppose that $F_{u} \neq 0$ and $\mathcal{F}=\{u=f(x, \varkappa)\}$ is a one-parametric family of solutions of (5). The derivative $f_{\varkappa}$ is nonzero since the parameter $\varkappa$ is essential. Therefore, $f_{12 \varkappa}=f_{\varkappa} F_{u}(f) \neq 0$. We express $\varkappa$ from the equality $u=f(x, \varkappa): \varkappa=\Phi(x, u)$ for some function $\Phi=\Phi(x, u)$ with $\Phi_{u} \neq 0$. Consider the operator $Q=\partial_{2}+\zeta \partial_{u}$, where the coefficient $\zeta=\zeta(x, u)$ is defined by the formula $\zeta=-\Phi_{2} / \Phi_{u} . Q[u]=0$ for any $u \in \mathcal{F}$. The ansatz $u=f(x, \varphi(\omega))$, where $\omega=x_{1}$, associated with $Q$, reduces (5) to the equation $\varphi_{\omega}=0$ since $f_{2 \varkappa} \neq 0$. Therefore [26], $Q$ is a reduction operator of equation (5) and hence the function $\zeta$ satisfies equation (6). Moreover, we have $\zeta_{u} \neq 0$ since otherwise the operator $Q$ would reduce (5) to an algebraic equation with respect to $\varphi$.

Corollary 8. Any adjoint singular reduction operators $Q=\partial_{2}+\zeta(x, u) \partial_{u}$ and $Q^{*}=\partial_{1}+$ $\zeta^{*}(x, u) \partial_{u}$ of equation (5) (where necessarily $\zeta_{u} \neq 0$ and $\zeta_{u}^{*} \neq 0$ ) are associated with the same one-parametric family of solutions of this equation.

Let $\zeta$ be an arbitrary solution of equation (6). Then $\zeta_{u} \neq 0$ and $Q=\partial_{2}+\zeta(x, u) \partial_{u}$ is a reduction operator of equation (5). Consider a one-parametric family $\mathcal{F}=\{u=f(x, \varkappa)\}$ of solutions of (5), which are invariant with respect to $Q$. (Such a family exists in view of Theorem 5.) Expressing the parameter $\varkappa$ from the equality $u=\tilde{f}(x, \varkappa)$, we obtain that $\varkappa=$ $\Phi(x, u)$, where $\Phi_{u} \neq 0 . \zeta=u_{2}=-\Phi_{2} / \Phi_{u}$ for any $u \in \mathcal{F}$, i.e., for any admissible values of $(x, \varkappa)$. This implies that the representation $\zeta=-\Phi_{2} / \Phi_{u}$ is true for any admissible value of $(x, u)$. This provides the background for the following statement.

Corollary 9. The nonlinear three-dimensional equation (66) is reduced by composition of the Bäcklund transformation $\zeta=-\Phi_{2} / \Phi_{u}, \zeta^{*}=-\Phi_{1} / \Phi_{u}$, where $\Phi$ is a function of $(x, u)$, and the hodograph transformation

the new independent variables: $\quad \tilde{x}_{1}=x_{1}, \quad \tilde{x}_{2}=x_{2}, \quad \varkappa=\Phi$, the new dependent variable: $\quad \tilde{u}=u$

to the equation (5) for the function $\tilde{u}=\tilde{u}(\tilde{x}, \varkappa)$ with $\varkappa$ playing the role of a parameter.

Proof. We take an arbitrary solution $\zeta$ of equation (6) (the condition $\zeta_{u} \neq 0$ is implicitly assumed to be satisfied) and set $\zeta^{*}=\left(F-\zeta_{1}\right) / \zeta_{u}$. In view of the Frobenius theorem, the system $\Phi_{2}+\zeta \Phi_{u}=0, \Phi_{1}+\zeta^{*} \Phi_{u}=0$ with respect to the function $\Phi=\Phi(x, u)$ is compatible since its compatibility condition $\zeta_{1}+\zeta^{*} \zeta_{u}=\zeta_{2}^{*}+\zeta \zeta_{u}^{*}$ coincides with (6) and hence is identically satisfied. We choose a nonconstant solution $\Phi$ of this system. Then $\Phi_{u} \neq 0, \zeta=-\Phi_{2} / \Phi_{u}$ and $\zeta^{*}=-\Phi_{1} / \Phi_{u}$. After the hodograph transformation, the latter equations take the form $\tilde{u}_{\tilde{x}_{2}}=\zeta(\tilde{x}, \tilde{u})$ and $\tilde{u}_{\tilde{x}_{1}}=\zeta^{*}(\tilde{x}, \tilde{u})$. This directly implies that for any value of $\varkappa$ the function $\tilde{u}=\tilde{u}(\tilde{x}, \varkappa)$ satisfies equation (5) . The parameter $\varkappa$ is essential in $\tilde{u}$ since $\tilde{u}_{\varkappa}=1 / \Phi_{u} \neq 0$.

It follows from the proof of Theorem 5 that the application of the inverse transformations to a one-parametric family of solutions of equation (5) results in a solution of equation (6). 
Note 4. For any equation from class (5) with $F_{u}=0$, reduction operators of the form $Q=$ $\partial_{2}+\zeta(x, u) \partial_{u}$, where $\zeta_{u} \neq 0$ (resp. $Q^{*}=\partial_{1}+\zeta^{*}(x, u) \partial_{u}$, where $\zeta_{u}^{*} \neq 0$ ) also are bijectively associated with one-parametric families of its solutions, having the form $\{u=f(x, \varkappa)\}$ where $f_{1 \varkappa} \neq 0$ (resp. $f_{2 \varkappa} \neq 0$ ). The one-parametric families with $f_{1 \varkappa}=0$ (resp. $f_{2 \varkappa}=0$ ) necessarily existing in this case correspond to ultra-singular reduction operators with $\zeta_{u}=0$ (resp. $\zeta_{u}^{*}=0$ ), and the correspondence is not one-to-one.

The above investigation of singular reduction operators of nonlinear wave equations of the form (5) shows that for these equations the natural partition of the corresponding sets of reduction operators is into triples of subsets singled out by the conditions
1) $\xi^{1}=0$
2) $\xi^{2}=0$
3) $\xi^{1} \xi^{2} \neq 0$.

After the factorization with respect to the equivalence relation of vector fields, we obtain three subsets of reduction operators, which have to be investigated separately. The defining conditions for these subsets are, respectively,
1) $\xi^{1}=0, \xi^{2}=1$
2) $\xi^{2}=0, \xi^{1}=1$
3) $\xi^{1} \neq 0, \xi^{2}=1$.

Since any equation from class (5) admits the point symmetry permuting $x_{1}$ and $x_{2}$, the second case is reduced to the first one and can be omitted. Finally we have two essentially different cases after factorization: the singular case $\xi^{1}=0, \xi^{2}=1$ and the regular case $\xi^{1} \neq 0, \xi^{2}=1$. The gauge $\xi^{2}=1$ is not uniquely possible in the regular case and may be varied for optimizing the further consideration of this case.

Consider the other standard form

$$
u_{11}-u_{22}=F(u)
$$

of nonlinear wave equations, obtained from (5) via the point transformation $\tilde{x}_{1}=x_{1}-x_{2}$, $\tilde{x}_{2}=x_{1}+x_{2}, \tilde{u}=u$. Using this transformation, all the results derived for class (5) can easily be extended to class (7). Thus, any equation of the form (7) possesses two singular sets of reduction operators, singled out by the conditions $\xi^{1}=-\xi^{2}$ and $\xi^{1}=\xi^{2}$, and one regular set of reduction operators, associated with the condition $\xi^{1} \neq \pm \xi^{2}$. The singular sets are mapped to each other by alternating the sign of $x_{2}$ and hence one of them can be excluded from the consideration. After factorization with respect to the equivalence relation of vector fields, we have two cases for our further study: the singular case $\xi^{1}=\xi^{2}=1$ and the regular case $\xi^{1} \neq \pm 1, \xi^{2}=1$.

For nonlinear wave equations of the general form

$$
u_{11}-\left(G(u) u_{2}\right)_{2}=F(u)
$$

where $G(u)>0$, the natural partitions of the sets of reduction operators are determined by more complicated conditions depending on the parameter-function $G$. We will not discuss these equation here. We only remark that the singular sets of the corresponding reduction operators are associated with the conditions $\xi^{2}=\sqrt{G} \xi^{1}$ and $\xi^{2}=-\sqrt{G} \xi^{1}$, respectively.

The above examples underline that the application of the conventional partition for factorization of sets of reduction operators often leads to the splitting of uniform cases and to combining essentially different ones. As a result, the derived systems of determining equations for the coefficients of reduction operators is far from optimal and difficult to investigate. Therefore, natural partitions based on taking into account the structure of singular families of reduction operators offers a decisive advantage. 


\section{Reduction operators and parametric families of solutions}

Proposition 4. Let $Q$ be a reduction operator of an equation $\mathcal{L}$. Then the weak singularity co-order of $Q$ for $\mathcal{L}$ equals the essential order of the corresponding reduced ordinary differential equation.

Proof. We carry out a point transformation in such a way that in the new variables the operator $Q$ has the form $Q=\partial_{x_{2}}$. (For convenience, for the new variables we use the same notations as for the old ones.) Then an ansatz constructed with $Q$ is $u=\varphi(\omega)$, where $\varphi=\varphi(\omega)$ is the new unknown function and $\omega=x_{1}$ is the invariant independent variable. The manifold $\mathcal{Q}_{(r)}$ is defined by the system $u_{\alpha}=0$, where $\alpha=\left(\alpha_{1}, \alpha_{2}\right), \alpha_{2}>0, \alpha_{1}+\alpha_{2} \leqslant r=\operatorname{ord} L$.

Since $Q \in \mathcal{Q}(\mathcal{L})$, there exist differential functions $\check{\lambda}=\check{\lambda}[\varphi]$ and $\check{L}=\check{L}[\varphi]$ of an order not greater than $r$ such that $\left.L\right|_{u=\varphi(\omega)}=\check{\lambda} \check{L}$ (cf. [26]). The function $\check{\lambda}$ does not vanish and may depend on $x_{2}$ as a parameter. The function $\check{L}$ is assumed to be of minimal order $\check{r}$ which may be attained up to the equivalence generated by nonvanishing multipliers. Then the reduced equation $\check{\mathcal{L}}: \check{L}=0$ has essential order $\check{r}$.

The condition $\operatorname{wsco}_{\mathcal{L}} Q=k$ means that there exists a strictly $k$ th order differential function $\tilde{L}=\tilde{L}[u]$ and a nonvanishing differential function $\tilde{\lambda}=\tilde{\lambda}[u]$ of an order not greater than $r$, which depend at most on $x$ and derivatives of $u$ with respect to $x_{1}$, such that $\left.L\right|_{\mathcal{Q}_{(r)}}=\left.\tilde{\lambda} \tilde{L}\right|_{\mathcal{Q}_{(r)}}$.

If $\check{r}$ would be less than $k$, we could use $\tilde{\lambda}_{\text {new }}=\left.\check{\lambda}\right|_{u \rightsquigarrow \varphi}$ and $\tilde{L}_{\text {new }}=\left.\check{L}\right|_{u \rightsquigarrow \varphi}$ in the definition of weak singularity and would arrive at the contradiction $\operatorname{wsco}_{\mathcal{L}} Q \leqslant$ ord $\tilde{L}_{\text {new }}=\check{r}<k$. Therefore, $\check{r} \geqslant k$. (Here, " $y \rightsquigarrow z$ " means that the value $y$ should be substituted instead of the value $z$.)

Suppose that $\check{r}>k$. We have the equality $\check{\lambda} \check{L}=\left.(\tilde{\lambda} \tilde{L})\right|_{u=\varphi(\omega)}$ in which the variable $x_{2}$ plays the role of a parameter. Fixing a value $x_{2}^{0}$ of $x_{2}$, we obtain the representation

$$
\check{L}=\left.\Lambda[\varphi] \tilde{L}\right|_{u=\varphi(\omega), x_{2}=x_{2}^{0}}, \quad \Lambda:=\left.\frac{\left.\tilde{\lambda}\right|_{u=\varphi(\omega)}}{\check{\lambda}}\right|_{x_{2}=x_{2}^{0}} \neq 0 .
$$

Since ord $\left.\tilde{L}\right|_{u=\varphi(\omega), x_{2}=x_{2}^{0}} \leqslant k<\check{r}$, this representation contradicts the condition that $\check{r}$ is the essential order of the reduced equation $\check{\mathcal{L}}$. Therefore, $\check{r}=k$. The inverse change of variables preserves the claimed property.

Corollary 10. Let $Q$ be a reduction operator of an equation $\mathcal{L}$. Then the weak singularity coorder of $Q$ for $\mathcal{L}$ equals the maximal number of essential parameters in families of $Q$-invariant solutions of $\mathcal{L}$.

Proof. The essential order $\check{r}$ of the reduced ordinary differential equation $\check{\mathcal{L}}$ associated with $Q$ coincides with the weak singularity co-order of $Q$ for $\mathcal{L}$. The maximal number of essential parameters in solutions of $\check{\mathcal{L}}$ equals the order of $\breve{\mathcal{L}}$. The substitution of these solutions into the corresponding ansatz leads to parametric families of $Q$-invariant solutions of $\mathcal{L}$, and all $Q$ invariant solutions of $\mathcal{L}$ are obtained in this way. Therefore, the maximal number of essential parameters in families of $Q$-invariant solutions of $\mathcal{L}$ equals $\check{r}$.

Corollary 11. Let $Q$ be a kth co-order weakly singular reduction operator of an equation $\mathcal{L}$. Suppose additionally that a differential function of minimal order, associated with $L$ on the manifold $\mathcal{Q}_{(r)}$ up to a nonvanishing multiplier, is of maximal rank in the derivative of $u$ of the highest order $k$ appearing in this differential function. Then $\mathcal{L}$ possesses a $k$-parametric family of $Q$-invariant solutions, and any $Q$-invariant solution of $\mathcal{L}$ belongs to this family.

Proof. Under this assumption, the reduced ordinary differential equation $\check{\mathcal{L}}$ associated with $Q$ can be written in normal form and hence has a $k$-parametric general solution which contains all solutions of $\check{\mathcal{L}}$. Substituting it into the corresponding ansatz, this solution gives a $k$-parametric family of $Q$-invariant solutions of $\mathcal{L}$. There are no other $Q$-invariant solutions of $\mathcal{L}$. 
Corollary 12. Suppose that a differential function of minimal order, associated with $L$ on the manifold $\mathcal{Q}_{(r)}$ up to a nonvanishing multiplier, is of maximal rank in the highest order derivative of $u$ appearing in this differential function. If the maximal number of essential parameters in families of $Q$-invariant solutions of $\mathcal{L}$ is not less than the weak singularity co-order of $Q$ for $\mathcal{L}$ then $Q$ is a reduction operator of $\mathcal{L}$.

Proof. Point transformations of the variables do not change the claimed property. We use the variables and notations from the proof of Proposition 4. Consider the differential function $\hat{L}[\varphi]=\left.\tilde{L}\right|_{u=\varphi(\omega)}$. It depends on $x_{2}$ as a parameter and ord $\hat{L}=k$. Due to the condition of maximal rank, we can resolve the equation $\hat{L}=0$ with respect to the highest order derivative $\varphi^{(k)}: \varphi^{(k)}=R[\varphi]$, where ord $R<k$.

If $R_{x_{2}} \neq 0$, splitting with respect to $x_{2}$ in the equation $\hat{L}=0$ results in an ordinary differential equation $\tilde{R}[\varphi]=0$ of an order lower than $k$. Any $Q$-invariant solution of $\mathcal{L}$ has the form $u=\varphi(\omega)$, where the function $\varphi$ satisfies, in particular, the equation $\tilde{R}[\varphi]=0$. This contradicts the condition that the maximal number of essential parameters in families of $Q$-invariant solutions of $\mathcal{L}$ is not less than $k$.

Therefore, $R_{x_{2}}=0$, i.e., the equation $\varphi^{(k)}=R[\varphi]$ is a reduced equation which is obtained from $\mathcal{L}$ by the substitution of the ansatz $u=\varphi(\omega)$ constructed with the operator $Q=\partial_{2}$.

Note 5. For any operator $Q$, the maximal number of essential parameters in families of $Q$ invariant solutions of $\mathcal{L}$ cannot be greater than $\operatorname{wsco}_{L} Q$.

Summing up the above consideration, we can formulate the following statement.

Proposition 5. Suppose that a differential function of minimal order, associated with the differential function $L[u]$ on the manifold $\mathcal{Q}_{(r)}(r=\operatorname{ord} L)$ up to a nonvanishing multiplier, is of maximal rank in the highest order derivative of $u$ appearing in this differential function. Then any two of the following properties imply the third one.

1) $Q$ is a reduction operator of the equation $\mathcal{L}: L=0$.

2) The weak singularity co-order of $Q$ for $\mathcal{L}$ equals $k(0 \leqslant k \leqslant r)$.

3) The equation $\mathcal{L}$ possesses a $k$-parametric family of $Q$-invariant solutions, and any $Q$ invariant solution of $\mathcal{L}$ belongs to this family.

The properties of ultra-singular vector fields as reduction operators are obvious.

Proposition 6. 1) Any ultra-singular vector field $Q$ of a differential equation $\mathcal{L}$ is a reduction operator of this equation. An ansatz constructed with $Q$ reduces $\mathcal{L}$ to the identity. Therefore, the family of $Q$-invariant solutions of $\mathcal{L}$ is parameterized by an arbitrary function of a single $Q$-invariant variable.

2) If the family of $Q$-invariant solutions of $\mathcal{L}$ is parameterized by an arbitrary function of a single $Q$-invariant variable then $Q$ is an ultra-singular vector field for $\mathcal{L}$.

\section{Reduction operators of singularity co-order 1}

Encouraged by the above investigation of evolution and, especially, wave equations, we study coorder one singular reduction operators of general partial differential equations in two independent and one dependent variables.

Consider an equation $\mathcal{L}: L=0$, where $L=L[u]$ is a differential function of order $r>1$. Suppose that the function $L$ admits a first co-order singular module of vector fields. (In view of Corollary 4, we can restrict ourselves to considering only strong singularity of vector fields for differential equations.) Without loss of generality, up to changing variables we can assume that the module contains a first co-order singular set $S=\left\{Q^{\zeta}\right\}$ of vector fields in reduced form, 
i.e., $Q^{\zeta}=\xi \partial_{1}+\partial_{2}+\zeta \partial_{u}$ for any smooth function $\zeta$ of $(x, u)$ and a fixed smooth function $\xi$. Additionally, we can assume $\xi \in\{0, u\}$.

By Theorem 1, the differential function $L$ can be written in the form $L=\check{L}\left(x, \Omega_{r, 1}\right)$, where

$$
\Omega_{r, 1}=\left(\omega_{\alpha}=D_{1}^{\alpha_{1}}\left(\xi D_{1}+D_{2}\right)^{\alpha_{2}} u, \alpha_{1} \leqslant 1, \alpha_{1}+\alpha_{2} \leqslant r\right),
$$

and $\check{L}_{\omega_{\alpha}} \neq 0$ for some $\omega_{\alpha}$ with $\alpha_{1}=1$. Then the restriction of $L$ to $\mathcal{Q}_{(r)}^{\zeta}$ coincides with the restriction, to the same manifold $\mathcal{Q}_{(r)}^{\zeta}$, of the function $\tilde{L}^{\zeta}=\check{L}\left(x, \tilde{\Omega}_{r, 1}\right)$, where

$$
\tilde{\Omega}_{r, 1}=\left(D_{1}^{\alpha_{1}}\left(Q^{\zeta}\right)^{\alpha_{2}} u, \alpha_{1} \leqslant 1, \alpha_{1}+\alpha_{2} \leqslant r\right) .
$$

Thus, the form of $\tilde{L}^{\zeta}$ is determined by the forms of $L$ and $\xi$ and a chosen value of the parameterfunction $\zeta$. Depending on the value of $\zeta$, the differential function $\tilde{L}^{\zeta}$ may either identically vanish or be of order 0 or 1 . This means that either the vector field $Q^{\zeta}$ is ultra-singular or $\operatorname{sco}_{L} Q^{\zeta}=0$ or $\operatorname{sco}_{L} Q^{\zeta}=1$, respectively. We investigate each of the above cases separately. Below we additionally suppose that the function $\tilde{L}^{\zeta}$ is of maximal rank with respect to $u$ (resp. $u_{1}$ ) if $\operatorname{sco}_{L} Q^{\zeta}=0$ (resp. $\left.\operatorname{sco}_{L} Q^{\zeta}=1\right)$.

The values of $\zeta$ for which $Q^{\zeta}$ for $\mathcal{L}$ is ultra-singular are singled out by the condition $\tilde{L}^{\zeta}=0$, where $u$ and $u_{1}$ are considered as independent variables. Splitting this condition with respect to $u_{1}$ gives a system $\mathcal{S}_{-1}$ of partial differential equations in $\zeta$ of orders less than $r$, which may be incompatible in the general case. The incompatibility of this system means that the set $S$ contains no ultra-singular vector fields. For example, evolution equations of orders greater than 1 and nonlinear wave equations of the form (5) with $F_{u} \neq 0$, in contrast to equations of the form (5) with $F_{u}=0$, have no ultra-singular vector fields, see Sections 5 and 6 , $\zeta$ satisfying the ultra-singularity condition guarantees that $Q^{\zeta} \in \mathfrak{Q}(\mathcal{L})$ and the family of $Q^{\zeta}$-invariant solutions of $\mathcal{L}$ is parameterized by an arbitrary function of a single $Q^{\zeta}$-invariant variable.

If $\operatorname{sco}_{L} Q^{\zeta}=0$, the parameter-function $\zeta$ satisfies the condition $\tilde{L}_{u_{1}}^{\zeta}=0$ with $u$ and $u_{1}$ viewed as independent variables, which is weaker than the ultra-singularity condition. Therefore, the corresponding system $\mathcal{S}_{0}$ of partial differential equations in $\zeta$ of orders less than $r$, obtained by splitting the zero co-order singularity condition with respect to $u_{1}$, has more chances of being compatible than $\mathcal{S}_{-1}$. Thus, any nonlinear wave equation of the form (5) with $F_{u} \neq 0$ admits zeroth co-order singular vector fields although this is not the case for ultra-singular vector fields. At the same time, evolution equations do not possess zeroth co-order singular vector fields.

Certain conditions which are sufficient for the compatibility of $\mathcal{S}_{0}$ can be formulated. Thus, if $\check{L}_{\omega_{(1,0)}}=0$ and $\xi_{u}=0$ then the system $\mathcal{S}_{0}$ is compatible since it is satisfied by any $\zeta$ with $\zeta_{u}=0$. In other words, $\operatorname{sco}_{L} Q^{\zeta} \leqslant 0$ for any $\zeta=\zeta(x)$. Let us consider this particular case in more detail. (Recall that under the condition $\xi_{u}=0$ the coefficient $\xi$ can be assumed, up to point transformations, to equal 0 but we will not use this possibility.)

If additionally $\check{L}_{\omega_{(0,0)}}=0$, the condition $\tilde{L}^{\zeta}=0$ under the assumption $\zeta=\zeta(x)$ implies only a single partial differential equation with respect to $\zeta$. Any of its solutions is a solution of $\mathcal{S}_{-1}$ and hence the corresponding vector field $Q^{\zeta}$ is ultra-singular for $L$.

Otherwise $\operatorname{sco}_{L} Q^{\zeta}=0$ and we can resolve the equation $\tilde{L}^{\zeta}=0$ with respect to $u$ : $u=G^{\zeta}(x)$, where the expression for the function $G^{\zeta}$ depends on the parameter-function $\zeta=\zeta(x)$ and its derivatives up to order $r-1$. Then the conditional invariance criterion is equivalent to the $r$ th order partial differential equation $\zeta=\xi G_{1}^{\zeta}+G_{2}^{\zeta}$ with respect to $\zeta$. If $\zeta$ is a solution of this equation then $Q^{\zeta}$ is a reduction operator of $\mathcal{L}$. The ansatz constructed with the operator $Q^{\zeta}$ can be taken in the form $u=\varphi(\omega)+G^{\zeta}(x)$, where $\varphi=\varphi(\omega)$ is the new unknown function and $\omega=\omega(x)$ is the invariant independent variable satisfying the equation $\xi \omega_{1}+\omega_{2}=0$. It reduces the initial equation $\mathcal{L}$ to a trivial algebraic equation $\varphi=0$, i.e., the function $u=G^{\zeta}(x)$ is a unique $Q^{\zeta}$-invariant solution of $\mathcal{L}$. Conversely, let us fix a solution $u=f(x)$ of the equation $\mathcal{L}$ and set $\zeta=\xi f_{1}+f_{2}$. Then $f=G^{\zeta}(x)$ and hence $\zeta=\xi G_{1}^{\zeta}+G_{2}^{\zeta}$, i.e., in view of the conditional 
invariance criterion $Q^{\zeta}=\xi \partial_{1}+\partial_{2}+\zeta \partial_{u}$ is a reduction operator of $\mathcal{L}$, and $\zeta_{u}=0$. The solution $u=f(x)$ is invariant with respect to $Q^{\zeta}$ by construction. Thus we obtain:

Theorem 6. Suppose that an equation $\mathcal{L}: L=0$ possesses a first co-order singular set $S=\left\{Q^{\zeta}\right\}$ of vector fields in reduced form $Q^{\zeta}=\xi \partial_{1}+\partial_{2}+\zeta \partial_{u}$ with $\xi_{u}=0$, i.e., its right hand side $L$ is represented in the form $L=\check{L}\left(x, \Omega_{r, 1}\right)$, where

$$
\Omega_{r, 1}=\left(\omega_{\alpha}=D_{1}^{\alpha_{1}}\left(\xi D_{1}+D_{2}\right)^{\alpha_{2}} u, \alpha_{1} \leqslant 1, \alpha_{1}+\alpha_{2} \leqslant r\right),
$$

$\check{L}_{\omega_{\alpha}} \neq 0$ for some $\alpha$ with $\alpha_{1}=1$, and additionally $\check{L}_{\omega_{(1,0)}}=0$ and $\check{L}_{\omega_{(0,0)}} \neq 0$. Then there exists a one-to-one correspondence between solutions of $\mathcal{L}$ and reduction operators from $S$ with $\zeta_{u}=0$. Namely, any such operator is of singularity co-order 0 and corresponds to the unique solution which is invariant with respect to this operator. The problems of solving the equation $\mathcal{L}$ and the exhaustive description of its reduction operators of the above form are completely equivalent.

Now we consider the regular values of $\zeta$ for which the singularity co-order of $Q^{\zeta}$ coincides with the singularity co-order of the whole family $S$ (and equals 1). If $\operatorname{sco}_{L} Q^{\zeta}=1$, the parameterfunction $\zeta$ satisfies the regularity condition $\tilde{L}_{u_{1}}^{\zeta} \neq 0$. Therefore, the equation $\tilde{L}^{\zeta}=0$ which is equivalent to $\mathcal{L}$ on the manifold $\mathcal{Q}_{(r)}^{\zeta}$ can be solved with respect to $u_{1}: u_{1}=G^{\zeta}(x, u)$, where the expression for the function $G^{\zeta}$ depends on the parameter-function $\zeta$ and its derivatives up to order $r-1$. Applied to the equation $\mathcal{L}$ and the operator $Q^{\zeta}$, the conditional invariance criterion implies only the equation

$$
\zeta_{1}+\zeta_{u} G^{\zeta}-\left(\xi_{1}+\xi_{u} G^{\zeta}\right) G^{\zeta}=\xi G_{1}^{\zeta}+G_{2}^{\zeta}+\zeta G_{u}^{\zeta}
$$

with respect to the function $\zeta$. Therefore, in this case the system of determining equations consists of the single equation (8) and, therefore, is not overdetermined. This equation can be rewritten as the compatibility condition

$$
\zeta_{1}+\zeta_{u} G^{\zeta}-\left(\xi_{1}+\xi_{u} G^{\zeta}\right) G^{\zeta}-\xi\left(G_{1}^{\zeta}+G_{u}^{\zeta} G^{\zeta}\right)=G_{2}^{\zeta}+\left(\zeta-\xi G^{\zeta}\right) G_{u}^{\zeta}
$$

of the equations $u_{1}=G^{\zeta}$ and $\xi u_{1}+u_{2}=\zeta$ with respect to $u$. The order of (8) equals $r$ and hence is greater than the order of the system $\mathcal{S}_{0}$. This guarantees (under certain conditions of smoothness, e.g., in the analytical case) that the equation (8) has solutions which are not solutions of $\mathcal{S}_{0}$. In other words, the equation $\mathcal{L}$ necessarily possesses first co-order singular reduction operators which belong to $S$.

The results of Section 7 imply that for each first co-order singular reduction operator $Q$ of the equation $\mathcal{L}$ there exist a one-parametric family of $Q$-invariant solutions of $\mathcal{L}$. If the equation $\mathcal{L}$ admits a co-order one singular module of vector fields, the converse statement is true as well.

Theorem 7. Suppose that an equation $\mathcal{L}: L=0$ possesses a co-order one singular set $S=\left\{Q^{\zeta}\right\}$ of vector fields in reduced form $Q^{\zeta}=\xi \partial_{1}+\partial_{2}+\zeta \partial_{u}$. Then for any one-parametric family $\mathcal{F}$ of solutions of $\mathcal{L}$ there exist a value of the parameter-function $\zeta=\zeta(x, u)$ such that $Q^{\zeta}$ is a reduction operator of $\mathcal{L}$ and each solution from $\mathcal{F}$ is invariant with respect to $Q^{\zeta}$.

Proof. Consider a one-parametric family $\mathcal{F}=\{u=f(x, \varkappa)\}$ of solutions of $\mathcal{L}$. The derivative $f_{\varkappa}$ is nonzero since the parameter $\varkappa$ is essential. From $u=f(x, \varkappa)$ we derive $\varkappa=\Phi(x, u)$ with some function $\Phi=\Phi(x, u)$, where $\Phi_{u} \neq 0$, and then define $\zeta=\zeta(x, u)$ by the formula

$$
\zeta=-\frac{\xi \Phi_{1}+\Phi_{2}}{\Phi_{u}}
$$

Since $f_{i}=-\left.\left(\Phi_{i} / \Phi_{u}\right)\right|_{u=f}, i=1,2$, then $\left.\xi\right|_{u=f} f_{1}+f_{2}=\left.\zeta\right|_{u=f}$, i.e., any solution from $\mathcal{F}$ is $Q^{\zeta}$-invariant. Then either $Q^{\zeta}$ is an ultra-singular vector field for $L$ or $\operatorname{sco}_{L} Q^{\zeta}=1$. (The case $\operatorname{sco}_{L} Q^{\zeta}=0$ is impossible since otherwise the equation $\mathcal{L}$ could not have a one-parametric family of $Q^{\zeta}$-invariant solutions.) Any ultra-singular vector field for $L$ is a reduction operator of $\mathcal{L}$. If $\operatorname{sco}_{L} Q^{\zeta}=1$ then $Q$ is a reduction operator of $\mathcal{L}$ in view of Corollary 12 . 
Corollary 13. Suppose that an equation $\mathcal{L}$ possesses a first co-order singular set $S=\left\{Q^{\zeta}\right\}$ of vector fields in reduced form $Q^{\zeta}=\xi \partial_{1}+\partial_{2}+\zeta \partial_{u}$, and that no element of $S$ is ultra-singular for $\mathcal{L}$. Then up to the equivalence of solution families there exists a bijection between oneparametric families of solutions of $\mathcal{L}$ and its first co-order singular reduction operators belonging to $S$. Namely, each operator of this kind corresponds to the family of solutions which are invariant under it. The problems of the construction of all one-parametric solution families of the equation $\mathcal{L}$ and the exhaustive description of its reduction operators of the above form are completely equivalent.

This bijection is broken in the presence of ultra-singular vector fields.

The above relation between one-parametric families of solutions and first co-order singular reduction operators can be stated as a connection between the initial equation $\mathcal{L}$ and the determining equation (8) .

Corollary 14. Suppose that an equation $\mathcal{L}: L=0$ possesses a first co-order singular set $S=$ $\left\{Q^{\zeta}\right\}$ of vector fields in reduced form $Q^{\zeta}=\xi \partial_{1}+\partial_{2}+\zeta \partial_{u}$. Then the determining equation for values of $\zeta$ corresponding to first co-order singular reduction operators of $\mathcal{L}$ is reduced by composition of the Bäcklund transformation

$$
\xi \Phi_{1}+\Phi_{2}+\zeta \Phi_{u}=0, \quad \Phi_{1}+G^{\zeta} \Phi_{u}=0
$$

where $\Phi$ is a function of $(x, u)$, and the hodograph transformation

$$
\begin{array}{ll}
\text { the new independent variables: } & \tilde{x}_{1}=x_{1}, \quad \tilde{x}_{2}=x_{2}, \quad \varkappa=\Phi, \\
\text { the new dependent variable: } & \tilde{u}=u
\end{array}
$$

to the initial equation $\mathcal{L}$ for the function $\tilde{u}=\tilde{u}(\tilde{x}, \varkappa)$ with $\varkappa$ playing the role of a parameter.

Proof. We fix an arbitrary solution $\zeta$ of equation (8), which additionally satisfies the condition $\tilde{L}_{u_{1}}^{\zeta} \neq 0$. In view of the Frobenius theorem, the equations $\xi \Phi_{1}+\Phi_{2}+\zeta \Phi_{u}=0$ and $\Phi_{1}+G^{\zeta} \Phi_{u}=0$ are compatible with respect to the function $\Phi=\Phi(x, u)$ since their compatibility condition coincides with (8) and hence is identically satisfied. We choose a nonconstant solution $\Phi$ of both these equations. Then $\Phi_{u} \neq 0$ and

$$
\zeta=-\xi \frac{\Phi_{1}}{\Phi_{u}}+\frac{\Phi_{2}}{\Phi_{u}}, \quad G^{\zeta}=-\frac{\Phi_{1}}{\Phi_{u}}
$$

After the hodograph transformation, the latter equations take the form $\xi \tilde{u}_{\tilde{x}_{1}}+\tilde{u}_{\tilde{x}_{2}}=\zeta(\tilde{x}, \tilde{u})$ and $\tilde{u}_{\tilde{x}_{1}}=G^{\zeta}(\tilde{x}, \tilde{u})$. This directly implies that for any value of $\varkappa$ the function $\tilde{u}=\tilde{u}(\tilde{x}, \varkappa)$ satisfies the equation $\mathcal{L}$. The parameter $\varkappa$ is essential in $\tilde{u}$ since $\tilde{u}_{\varkappa}=1 / \Phi_{u} \neq 0$.

It follows from the proof of Theorem 7 that the application of the inverse transformations to a one-parametric family of solutions of the initial equation $\mathcal{L}$ results in a solution of equation (8) if the defined value of $\zeta$ satisfies the regularity condition $\tilde{L}_{u_{1}}^{\zeta} \neq 0$.

\section{Acknowledgements}

The authors are grateful to Vyacheslav Boyko for useful discussions and interesting comments. MK was supported by START-project Y237 of the Austrian Science Fund. The research of ROP was supported by the Austrian Science Fund (FWF), project P20632. 


\section{References}

[1] Arrigo D.J., Hill J.M., Broadbridge P., Nonclassical symmetry reductions of the linear diffusion equation with a nonlinear source, IMA J. Appl. Math., 1994, 52, 1-24.

[2] Bila N. and Niesen J., On a new procedure for finding nonclassical symmetries, J. Symbolic Comput., 2004, 38, 1523-1533.

[3] Bluman G.W. and Cole J.D., The general similarity solution of the heat equation, J. Math. Mech., 1969, 18, 1025-1042.

[4] Clarkson P.A. and Kruskal M.D., New similarity solutions of the Boussinesq equation, J. Math. Phys., 1989, 30, 2201-2213.

[5] Clarkson P.A. and Mansfield E.L., Symmetry reductions and exact solutions of a class of nonlinear heat equations, Physica D, 1994, 70, 250-288.

[6] Clarkson P.A. and Mansfield E.L., Algorithms for the nonclassical method of symmetry reductions, SIAM J. Appl. Math. 1994, 54, 1693-1719.

[7] Fushchych W.I. and Popowych R.O., Symmetry reduction and exact solution of the Navier-Stokes equations. I, J. Nonlinear Math. Phys, 1994, 1, 75-113.

[8] Fushchych W.I., Serov N.I., The symmetry and some exact solutions of the nonlinear many-dimensional Liouville, d'Alembert and eikonal equations, J. Phys. A: Math. Gen., 1983, 16, 3645-3658.

[9] Fushchych W.I., Shtelen W.M. and Serov N.I., Symmetry analisys and exact solutions of equations of nonlinear mathimatical physics, Dordrecht, Kluwer Academic Publishers, 1993.

[10] Fushchych W. I., Shtelen W.M., Serov M.I. and Popovych R.O., Q-conditional symmetry of the linear heat equation, Proc. Acad. Sci. Ukraine, 1992, no. 12, 28-33.

[11] Fushchych W.I. and Tsyfra I.M., On a reduction and solutions of the nonlinear wave equations with broken symmetry, J. Phys. A: Math. Gen., 1987, 20, L45-L48.

[12] Fushchych W.I. and Zhdanov R.Z., Conditional symmetry and reduction of partial differential equations, Ukr. Math. J., 1992, 44, 970-982.

[13] Mansfield E.L., The nonclassical group analysis of the heat equation, J. Math. Anal. Appl., 1999, 231, $526-542$.

[14] Olver P., Applications of Lie groups to differential equations, New-York, Springer-Verlag, 1993.

[15] Olver P., Direct reduction and differential constraints, Proc. R. Soc. Lond. A, 1994, 444, 509-523.

[16] Olver P.J. and Vorob'ev E.M., Nonclassical and conditional symmetries, in CRC Handbook of Lie Group Analysis of Differential Equations, Vol. 3, Editor N.H. Ibragimov, Boca Raton, Florida, CRC Press, 1996, 291-328.

[17] Ovsiannikov L.V., Group analysis of differential equations, New York, Academic Press, 1982.

[18] Popovych R.O., On the symmetry and exact solutions of a transport equation, Ukr. Math. J., 1995, 47, $142-148$.

[19] Popovych R.O., On a class of $Q$-conditional symmetries and solutions of evolution equations, in Symmetry and Analytic Methods in Mathematical Physics, Proceedings of Institute of Mathematics, Kyiv, 1998, 19, 194-199 (in Ukrainian).

[20] Popovych R.O., Equivalence of $Q$-conditional symmetries under group of local transformation, in Proceedings of the Third International Conference "Symmetry in Nonlinear Mathematical Physics" (Kyiv, July 1218, 1999), Proceedings of Institute of Mathematics, Kyiv, 2000, 30, Part 1, 184-189; arXiv:math-ph/0208005.

[21] Popovych R.O., Reduction operators of linear second-order parabolic equations, J. Phys. A, 2008, 41, 185202; arXiv:0712.2764

[22] Popovych R.O., Vaneeva O.O and Ivanova N.M., Potential nonclassical symmetries and solutions of fast diffusion equation, Phys. Lett. A, 2007, 362, 166-173; arXiv:math-ph/0506067

[23] Vasilenko O.F. and Popovych R.O., On class of reducing operators and solutions of evolution equations, Vestnik PGTU, 1999, 8, 269-273 (in Russian).

[24] Webb G.M., Lie symmetries of a coupled nonlinear Burgers-heat equation system, J. Phys. A: Math. Gen., 1990, 23, 3885-3894.

[25] Zhdanov R.Z. and Lahno V.I., Conditional symmetry of a porous medium equation, Phys. D, 1998, 122, 178-186.

[26] Zhdanov R.Z., Tsyfra I.M. and Popovych R.O., A precise definition of reduction of partial differential equations, J. Math. Anal. Appl., 1999, 238, 101-123; arXiv:math-ph/0207023. 